\title{
Time-Resolved Study of ICD in Ne Dimers Using FEL Radiation
}

K. Schnorra,*, A. Senftleben ${ }^{\text {b }}$, G. Schmid ${ }^{\mathrm{a}}$, S. Augustin ${ }^{\mathrm{a}}$, M. Kurka ${ }^{\mathrm{a}}$, A. Rudenko ${ }^{\text {c }}$, L. Foucar ${ }^{\text {d }}$, A. Broska $^{\mathrm{a}}$, K. Meyer $^{\mathrm{a}}$, D. Anielski ${ }^{\mathrm{e}}$, R. Bolle, D. Rolles ${ }^{\mathrm{c}}$, M. Kübel ${ }^{\mathrm{f}}$, M.F. Kling ${ }^{\mathrm{f}}$, Y.H. Jiang ${ }^{\mathrm{g}}$, S. Mondal ${ }^{\mathrm{h}}$, T. Tachibana $^{\mathrm{h}}$, K. Ueda ${ }^{\mathrm{h}}$, T. Marchenko ${ }^{\mathrm{i}}$, M. Simon ${ }^{\mathrm{i}}$, G. Brenner ${ }^{\mathrm{e}}$, R. Treusch ${ }^{\mathrm{e}}$, S. Scheit ${ }^{\mathrm{j}}$, V. Averbukh ${ }^{\mathrm{k}}$, J. Ullrich ${ }^{1}$, T. Pfeifer ${ }^{\mathrm{a}}$, C.D. Schröter ${ }^{\mathrm{a}}$, R. Moshammer ${ }^{\mathrm{a}, *}$

\author{
${ }^{a}$ Max-Planck-Institut für Kernphysik, Saupfercheckweg 1, 69117 Heidelberg, Germany \\ ${ }^{b}$ Universität Kassel, 34132 Kassel, Germany \\ ${ }^{c}$ J.R. Macdonald Laboratory, Kansas State University, Manhattan, Kansas 66506, USA \\ ${ }^{d}$ Max-Planck-Institut für medizinische Forschung, 69120 Heidelberg, Germany \\ ${ }^{e}$ Deutsches Elektronen-Synchrotron, 22607 Hamburg, Germany \\ ${ }^{f}$ Physics Department, Ludwig-Maximilians-Universität München, 85748 Garching, Germany \\ ${ }^{g}$ Shanghai Advanced Research Institute, Chinese Academy of Sciences, Shanghai 201210, China \\ ${ }^{h}$ Institute of Multidisciplinary Research for Advanced Materials, Tohoku University, Sendai 980-8577, Japan \\ ${ }^{i}$ Laboratoire de Chimie Physique-Matière et Rayonnement, UPMC and CNRS, 75231 Paris, France \\ ${ }^{j}$ Goethe-Universität, 60438 Frankfurt, Germany \\ ${ }^{k}$ Imperial College London, London SW7 2AZ, United Kingdom \\ ${ }^{l}$ Physikalisch-Technische Bundesanstalt, 38116 Braunschweig, Germany
}

\begin{abstract}
Interatomic Coulombic Decay (ICD) is a relaxation phenomenon, which takes place in weakly bound atomic and molecular systems, typically within a few to hundreds of femtoseconds depending on the system and the particular decay mechanism. The creation of ICD-active states requires the production of highly excited systems, usually populated by innershell ionization or excitation. To this end, XUV and X-ray radiation from synchrotrons was conventionally applied for the majority of experiments due to the desired state-selective ionization of certain sub-shells. The advent of Free-Electron Lasers (FELs) has enabled an entirely new class of experiments, which finally allow to trace ICD directly in the time domain due to the femtosecond pulse duration. Within this paper, the first time-resolved ICD measurement using an XUV-pump-XUV-probe scheme will be discussed in detail. The experiment was performed on neon dimers and ICD was triggered by removing a $2 s$ electron from one of the neon atoms using a $58 \mathrm{eV}$ pulse from the FEL in Hamburg (FLASH). The onset of ICD was probed with a delayed copy of the trigger pulse that further ionized one of the two $\mathrm{Ne}^{+}$ions emerging after ICD. Thus, the delay-dependent yield of coincident $\mathrm{Ne}^{+}+\mathrm{Ne}^{2+}$ ion pairs contains the lifetime of the $2 s$-innershell vacancy decaying via ICD. The result of $150 \mathrm{fs} \pm 50 \mathrm{fs}$ is in good agreement with theory but only for those calculations that explicitly take nuclear motion into account.
\end{abstract}

Keywords: ICD, FEL, time resolved

PACS: 36.40.Mr, 33.80.Eh, 41.60.Cr, 82.33.Fg

\section{Contents}

\section{Introduction}

\section{Experiment}

\section{Results}

3.1 Pump-Probe Scheme . . . . . . . . . . . . 3

3.2 Classical Simulations . . . . . . . . . . . . 5

3.3 Lifetime Determination . . . . . . . . . . . 7

3.4 Competing Pathways . . . . . . . . . . . 8

3.5 Simulation of Competing Processes . . . . . 9

3.6 Comparison with Theory . . . . . . . . . 11

\section{Discussion}

\footnotetext{
${ }^{*}$ Corresponding authors

Email addresses: kirsten.schnorr@mpi-hd.mpg.de (K. Schnorr), robert.moshammer@mpi-hd.mpg.de (R. Moshammer)
}

5 Conclusion and Outlook

5

(

8

11

\section{Introduction}

Interatomic and Intermolecular Coulombic Decay (ICD) are ultrafast relaxation mechanisms on the few to hundred femtosecond (fs) time-scale [1] where an excited atom or molecule relaxes by transferring its excess energy to a neighbor, which is in turn ionized (cf. Fig. 1). It appears in various environments, like clusters [2, 3], He droplets [4], fullerenes [5], and aqueous solution [6]. Therefore, ICD is expected to be a universal relaxation mechanism in weakly bound systems. It is of particular relevance for biological samples because the secondarily emitted ICD electrons have typically low kinetic energies and therefore damage molecular bonds efficiently by dissociative electron attachment [7]. As a consequence, ICD is expected to play an important role for DNA damage in radiation therapy [8] and may even be exploited as a tool to destroy malign tis- 


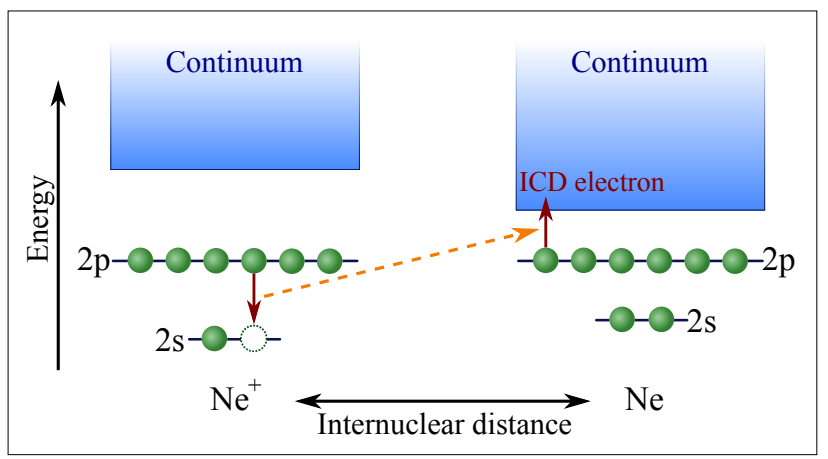

Figure 1: ICD in $\mathrm{Ne}_{2}$ : A previously innershell-ionized $\mathrm{Ne}^{+}$ion relaxes via ICD by refilling its $2 s$ vacancy and transferring its excess energy to a neighboring $\mathrm{Ne}$ atom, which is in turn ionized.

sue $[9,10]$. However, only little is known experimentally about such fundamental property of this decay mechanism as the decay time.

The lifetimes $\tau$ of non-local relaxation mechanisms such as ICD and the related Electron-Transfer-Mediated Decay (ETMD) [11], are challenging to study because electronic and nuclear degrees of freedom are often strongly interwoven. Consequently, the decay widths $\Gamma=1 / \tau$ exhibit a complex $R$ dependent behavior, where $R$ is the internuclear distance. Theoretically this challenge is tackled by calculating $\Gamma$ for different $R$, as shown in Fig. 2 [12] and adding nuclear wave-packet dynamics with $\Gamma(R)$ [13]. This approach has been successfully demonstrated for rare-gas dimers, like $\mathrm{Ne}_{2}$ [14], but it is too expensive from a computational point of view for complex molecular systems. In particular for larger systems, the need to include nuclear dynamics into the calculations is debated. As the number of atomic or molecular neighbors grows, the ICD lifetimes decrease [15]: While an isolated excited $\mathrm{Ne}^{+}\left(2 s^{-1}\right)$ ion needs about $0.2 \mathrm{~ns}$ [16] to return to its ground state via radiative decay, the same ion embedded into a large neon cluster relaxes within a few fs $[15,17]$ via ICD. The theoretical prediction of only $2 \mathrm{fs}$ for the decay of a neon ion caged into a fullerene is even faster [5]. ICD in large water clusters [18] and dimers [19] was experimentally confirmed to take place within a few to ten fs. Therefore, a fast electronic decay on the few fs time-scale might not be affected by the onset of nuclear motion, which typically occurs within tens of fs. On the other hand, for more neighbors and consequently faster decays ICD takes place on the time-scales of Auger decay and autoionization. Thus, ICD might play an essential role in particular in large systems because it is not outpaced by competing processes. In addition, it was demonstrated that nuclear motion has a significant impact on the decay mechanism even if the ICD lifetime is only a few fs: By a combined experimental and theoretical investigation on the core-ionization dynamics of liquid water, it was shown that proton transfer clearly affects the ultrafast electronic relaxation mechanism [20]. Very recently, calculations revealed that the onset of proton transfer in a water environment can significantly enhance non-local relaxation processes, like ICD and ETMD

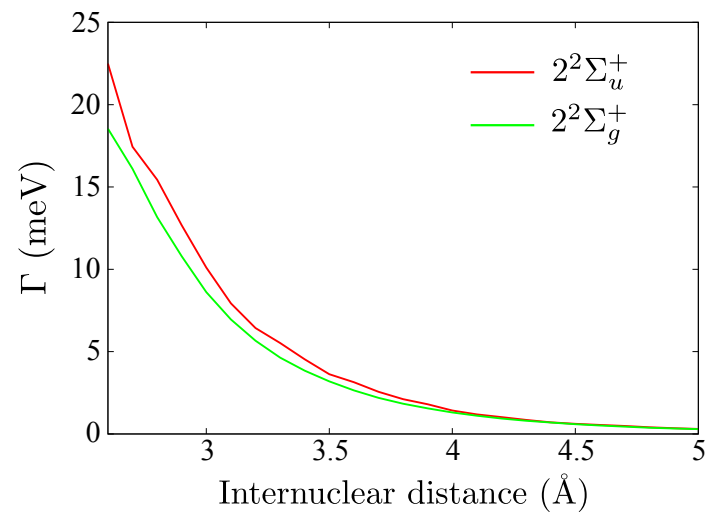

Figure 2: Calculated decay widths $\Gamma$ for ICD in $\mathrm{Ne}^{+}\left(2 s^{-1}\right)$ for both intermediate states, $2^{2} \Sigma_{g}^{+}$and $2^{2} \Sigma_{u}^{+}$. The electronic decay width was calculated at five internuclear distances using the Fano-ADC method [12] and interpolated in between.

[21]. Therefore, despite the common belief, nuclear motion is not negligible for large systems due to the fast proton-transfer dynamics.

So far, the majority of ICD experiments were performed using synchrotrons. The state-selectivity of XUV and X-ray radiation is ideal for the removal of certain innershell electrons and creating ICD-active states. However, synchrotrons deliver pulses with picosecond duration, which is by far not sufficient to resolve ICD directly in the time-domain. Therefore, temporal information on ICD had to be extracted indirectly in the frequency domain. An early experiment determined the ICD lifetime of the $2 s$ innershell vacancy in large neon clusters by measuring the width of the photoemission line. For bulk atoms, $6 \mathrm{fs}$ were extracted while vacancies in surface atoms were found to decay within $30 \mathrm{fs}$ [17]. However, the width of the photoline returns the lifetime only if nuclear motion during the decay process is negligible. For the bulk atoms of large neon clusters this assumption is justified. For small systems, like dimers or light molecules, in particular those containing hydrogen, nuclear motion cannot be neglected. However, one exception should be pointed out: If only one specific vibrational level of an ICDactive state is populated, the state is stationary and therefore the decay width independent of $R$, as shown in [22, 23]. A recent experiment succeeded in investigating the impact of nuclear motion on the ICD lifetime by means of He-dimers exploiting Post-Collision Interaction (PCI) [24, 25]. Temporal information was extracted by converting electron kinetic energies in time delays using a classical model [26]. In summary, all of these methods required a transformation from the frequency- to the time-domain which implies certain assumptions about the decay mechanism.

This paper will focus on the first ICD experiment performed directly in the time-domain on neon dimers employing an XUVpump-XUV-probe scheme at the Free-Electrons Laser (FEL) in Hamburg (FLASH) [27]. Only recently, fast-pulsed and short-wavelength light sources based on High-Harmonic Generation (HHG) and FELs became available. They opened up the possibility to investigate dynamics of innershell-ionized or 
-excited systems on the fs time-scale. HHG sources and FELs offer ideal conditions to study ICD because they combine the state-selectivity of XUV radiation and X-rays with the temporal resolution to access ultrafast molecular dynamics in real time. While HHG sources stand out thanks to their pulse durations down to the attosecond time-domain and their excellent synchronization with the optical driving laser, FELs feature orders of magnitude higher intensities from the XUV into the X-ray regime. For the latter, pulse durations of a few fs were demonstrated in the X-ray regime by the Linac Coherent Light Source (LCLS) [28] and tens of fs in the XUV energy regime are routinely available at FLASH. For pump-probe experiments these pulses may either be used with optical lasers that can be synchronized up to a few fs [29] or with a delayed replica split-off from the XUV or X-ray pulse itself. In addition, the high intensities of FELs allow to study collective relaxation dynamics in clusters, which require the simultaneous excitation of multiple atoms [30, 31].

Within this paper, we will discuss the ICD-lifetime determination in $\mathrm{Ne}_{2}$ using an XUV-pump-XUV-probe scheme. In Sec. 2, the experimental set-up, consisting of a Reaction Microscope in combination with a split- and delay-mirror set-up, will be covered. Sec. 3 will introduce the employed pumpprobe scheme with emphasis on the relevant potential-energy curves (PECs) of the neon dimer in Sec. 3.1. In order to interpret the obtained experimental data, classical simulations were performed that will be discussed in Sec. 3.2. Based on these results, the lifetime determination procedure will be discussed in Sec. 3.3. Since the lifetime determination relies on monitoring the delay-dependent ion yield of a specific coincidence channel, it is crucial to investigate competing pathways that could also feed into this channel. Possible background sources and their impact on the lifetime determination will be discussed in Sec. 3.4. In Sec. 4, the experimental data will be compared with the outcome of different calculations. Finally, the paper closes in Sec. 5 with a discussion of the obtained results.

\section{Experiment}

The experiment was carried out at the unfocused branch of beamline BL3 at FLASH [32] using a Reaction Microscope (REMI) [33] with XUV split- and delay-unit. The FEL delivered 800 pulses/s at an intensity of roughly $10^{12} \mathrm{~W} / \mathrm{cm}^{2}$, which was chosen comparatively low to suppress multi-photon ionization within a single pulse. The central photon energy of the FEL was $58.2 \mathrm{eV}$ with a bandwidth of roughly $1 \mathrm{eV}$ (FWHM) due to SASE fluctuations [34]. As depicted in Fig. 3, the incoming pulse of roughly $60 \mathrm{fs}$ (FWHM) passes through the interaction region onto a 1 inch back-reflecting $\mathrm{Mo} / \mathrm{Si}$ multi-layer mirror that is split in half. By moving one half of the mirror back and forth with respect to the other, a temporal delay between the geometrically split pulses is introduced. Both beams are then spatially overlapped in the focal point, which has a diameter of $10 \mu \mathrm{m}-$ alsoload $15 \mu \mathrm{m}$. The focus is located within the gas jet created by supersonic expansion. To this end, $\mathrm{Ne}$ gas is expanded through a $30 \mu \mathrm{m}$ diameter nozzle, kept at room temperature, with an injection pressure of 21 bar. The expansion

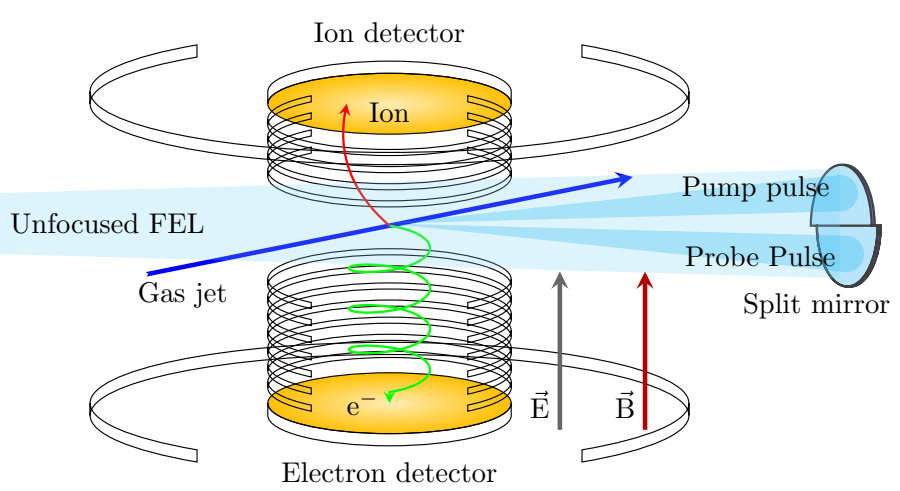

Figure 3: Sketch of the REMI with split- and delay-mirror set-up: The unfocussed FEL beam passes through the interaction chamber onto a cut-in-half back-reflecting multilayer mirror, which focuses the split beams into the supersonic gas jet. Charged fragments are guided onto the time- and positionsensitive detectors on the top and bottom by means of homogeneous electric and magnetic fields.

conditions were optimized to suppress the production of clusters larger than $\mathrm{Ne}_{2}$ [35]. The exact internal temperature of the jet could not be determined during the measurement, however comparison to earlier experiments shows that the dimers were most likely produced in their vibrational ground state. The momenta and energies of all ions were reconstructed by means of a REMI. A homogenous electric field of $35 \mathrm{~V} / \mathrm{cm}$ was applied to accelerate the ions over a distance of $10.5 \mathrm{~cm}$ from the interaction region onto a time- and position-sensitive detector (MCP and delayline anode with $120 \mathrm{~mm}$ diameter active area). From the impact time and position the three-dimensional momenta were reconstructed.

\section{Results}

\subsection{Pump-Probe Scheme}

The XUV-pump-XUV-probe scheme used to determine the ICD lifetime in $\mathrm{Ne}_{2}$ is illustrated in Fig. 4. For its explanation, we introduce the relevant PECs in $\mathrm{Ne}_{2}$ that are accessible via two-photon absorption from the ground state. The energy region between $52 \mathrm{eV}$ and $90 \mathrm{eV}$ contains further PECs, which are not displayed here for simplicity.

The role of the pump pulse is to initiate ICD by the creation of a $2 s$ inner-valence vacancy at one of the neon atoms. Once the excited $\mathrm{Ne}_{2}^{+}\left(2 s^{-1}\right)$ state is created, the essential question arises how long it takes until the excited dimer decays into $\mathrm{Ne}^{+}-\mathrm{Ne}^{+}$ions. In order to answer this question, the dimer's charge state has to be monitored continuously. Experimentally, this is realized by probing the charge state with a delayed second XUV pulse that further ionizes the dimer into a distinct state, which reveals whether the dimer had already decayed by the time the probe pulse arrived or not. Thus, by scanning the time delay and simultaneously detecting the fragments created after the probe pulse, the lifetime of the excited state, i.e., the ICD lifetime, is determined. Fig. 4 shows a simplified version 


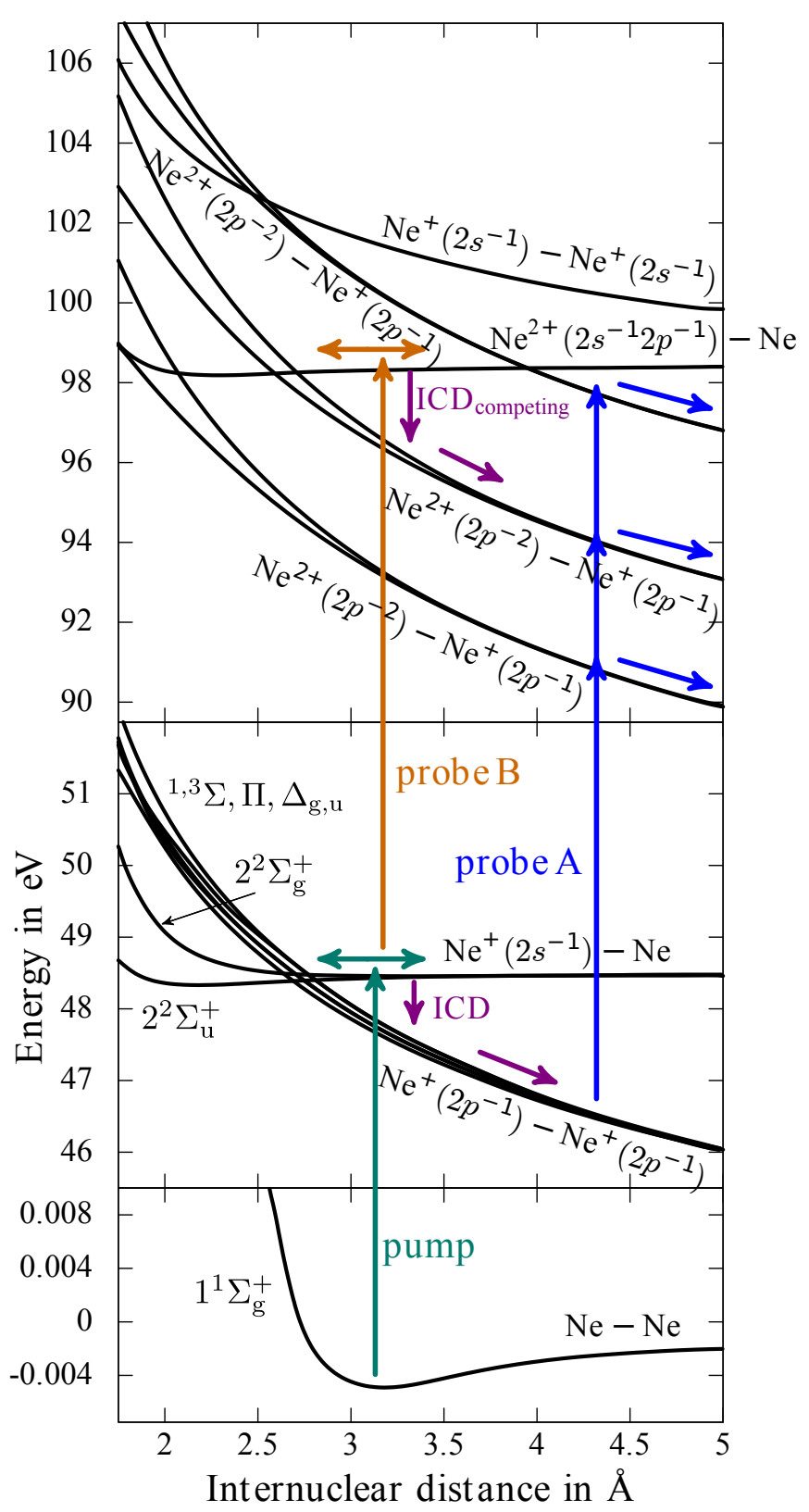

Figure 4: Relevant PECs for the illustration of the pump-probe scheme for the lifetime determination. A preceding photon from the pump pulse initiates ICD by the creation of a $2 s$ vacancy at one of the neon atoms. If ICD has occurred before the probe pulse arrives (probe A), one of the $\mathrm{Ne}^{+}-\mathrm{Ne}^{2+}$ curves is accessed. For an early probe pulse (probe $\mathrm{B}$ ), the unstable $\mathrm{Ne}^{2+}\left(2 s^{-1} 2 p^{-1}\right)-\mathrm{Ne}$ state may be populated, which decays via a competing $\mathrm{ICD}_{\text {competing into }} \mathrm{Ne}^{+}-\mathrm{Ne}^{2+}$, as will be discussed in Sec. 3.4. The PECs were extracted from Ref. [36]. For each curve the final charge state in the dissociation limit is indicated at the respective line.
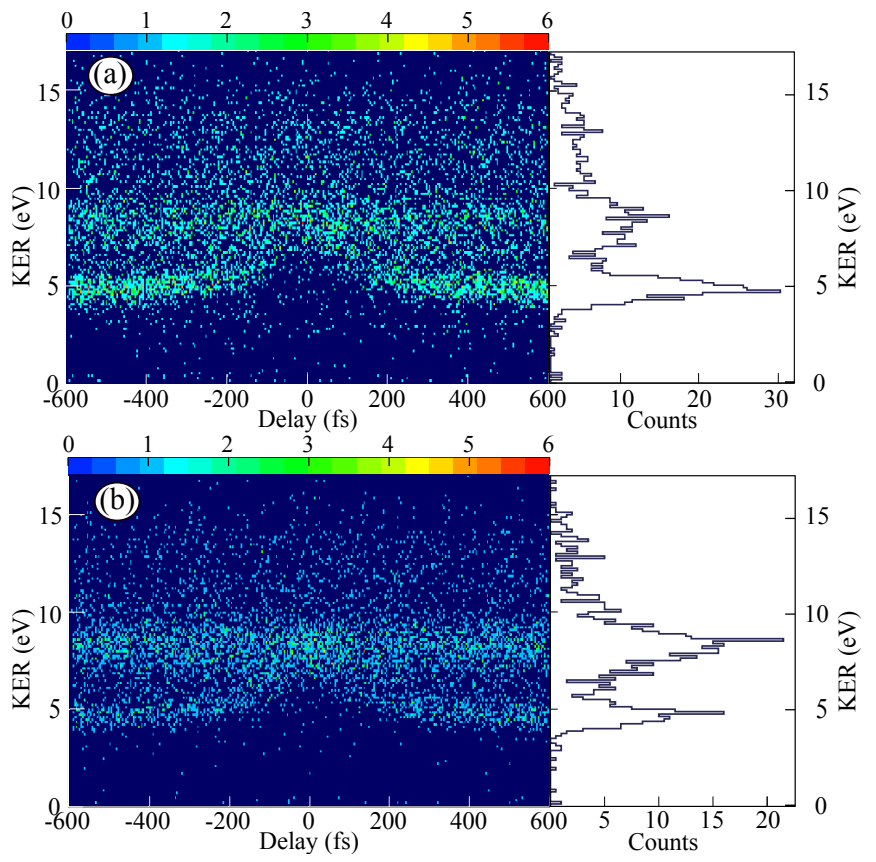

Figure 5: Delay-dependent KER spectra of coincident $\mathrm{Ne}^{+}-$ $\mathrm{Ne}^{2+}$ fragments for lower FEL intensity in (a) and a higher one in (b). In addition the projections onto the KER axis for large delays are shown in the right panels. Note that the multi-photon contribution around $8 \mathrm{eV}$ is pronounced for higher photon intensity in (b), while the pump-probe contribution around $5 \mathrm{eV}$ is dominating for the lower intensity in (a). The total ion yield in (b) is lower due to shorter measurement times. Therefore, different scales were chosen for the projections of (a) and (b).

of the pump-probe scheme: If ICD has occurred by the time the probe pulse arrives, one of the two created $\mathrm{Ne}^{+}$fragments is further ionized, resulting in a coincident $\mathrm{Ne}^{+}-\mathrm{Ne}^{2+}$ ion pair. If instead ICD has not taken place when the probe pulse impinges, a further electron from the dimer ion $\mathrm{Ne}_{2}^{+}\left(2 s^{-1}\right)$ is removed, resulting in $\mathrm{Ne}_{2}^{2+}$. This molecular ion will most likely dissociate into $\mathrm{Ne}-\mathrm{Ne}^{2+}$ or $\mathrm{Ne}^{+}-\mathrm{Ne}^{+}$, as will be discussed in Sec. 3.4 in more detail. Thus, a PEC with the final charge state $\mathrm{Ne}^{+}-\mathrm{Ne}^{2+}$ is only reachable for those dimers that have already undergone ICD before the probe pulse arrives. Therefore, we expect a time-dependent $\mathrm{Ne}^{+}-\mathrm{Ne}^{2+}$ ion yield that increases as according to the ICD lifetime. However, these considerations do only take into account the situation in which a single photon is absorbed within the pump and a single photon within the probe pulse.

Since the lifetime of the $2 s$ vacancy in $\mathrm{Ne}_{2}^{+}$is determined by inspection of the yield of coincident $\mathrm{Ne}^{+}-\mathrm{Ne}^{2+}$ ion pairs, all possible pathways leading to this fragmentation channel must be analyzed carefully. The delay-dependent KER of coincident $\mathrm{Ne}^{+}-\mathrm{Ne}^{2+}$ fragments for a data set recorded at low intensity is shown in Fig. 5(a), as well as another one recorded at higher intensity in Fig. 5(b). During the measurement, the delay between pump and probe pulse was continuously scanned between $-600 \mathrm{fs}$ and $600 \mathrm{fs}$ (cf. Fig. 5), meaning one of the pulses is preceding the other for negative delays and succeeding it for 
positive delays. Therefore, the recorded data are mirror symmetric with respect to time delay zero where both pulses overlap temporally. The spectra show two dominant features: one with a constant KER around $8.6 \mathrm{eV}$ and a time-dependent one converging to $5 \mathrm{eV}$ for large delays. The time-independent band stems from $\mathrm{Ne}^{+}-\mathrm{Ne}^{2+}$ ions that were created by multi-photon absorption within one pulse, which is confirmed by evaluating the KER as follows. For two point-like charged particles, one singly and the other doubly charged, the expected KER assuming an instantaneous Coulomb-explosion at the equilibrium internuclear distance of $R_{\text {eq }}=3.1 \AA$ amounts to $9.28 \mathrm{eV}$. Since this value is close to the observed $8.6 \mathrm{eV}$, a relatively fast ionization process can be assumed. In order to create the triply ionized dimer $\mathrm{Ne}_{2}^{3+}$ directly from the ground state, three photons must be absorbed. This assumption is supported by the KER distributions of the high and the low intensity spectra in Fig. 5: At the higher intensity, the peak at $8.6 \mathrm{eV}$, which is attributed to three-photon absorption, is much more pronounced. As our pump-probe scheme relies on single-photon absorption within the pump and the probe, contributions from multi-photon processes cause a constant background to the delay-dependent ICD signal. Thus, in order to achieve a good contrast of signal to background, multi-photon processes were suppressed by keeping a low FEL intensity of roughly $10^{12 \mathrm{~W}} / \mathrm{cm}^{2}$, for which data is shown in Fig. 5(a). Here, the three-photon contribution is clearly reduced compared to the delay-dependent feature. We would like to emphasize, that multi-photon contributions only cause a constant offset in the ion yield, but do not affect our lifetime result.

The lifetime information is contained in the time-dependent trace in Fig.5. For small delays, the constant and the time-dependent contributions overlap energetically because the steepest PEC, $\mathrm{Ne}^{+}-\mathrm{Ne}^{2+}$, is accessed around $R_{\mathrm{eq}}$. Instead, for larger delays an intermediate charge state with a shallower PEC is populated, which results in a lower KER because the final potential curve is accessed at an increased internuclear distance. For asymptotically large delays and correspondingly large internuclear distances, no energy is gained at all by the population of a steeper curve because from some distance on all potentials can be considered flat. As a result we are able to reconstruct the intermediate charge state by analyzing the asymptotic KER for large delays. As it converges to an energy of roughly $5 \mathrm{eV}$, we conclude that the precursor $\mathrm{Ne}^{+}-\mathrm{Ne}^{+}$was populated by the pump pulse, which is in line with the proposed scheme in Fig. 4. The observed KER is slightly higher than the expected one of $4.64 \mathrm{eV}$ from a Coulomb explosion at $R_{\text {eq }}$. This indicates that the KER has not yet converged to its asymptotic value after the observed $600 \mathrm{fs}$. Around time delay zero the ion yield is increased due to the doubled intensity in the temporal overlap region of the pulses. At high intensities, the multi-photon process becomes more likely, resulting in an increased number of $\mathrm{Ne}^{+}-\mathrm{Ne}^{2+}$ ions. The enhanced ion yield around time delay zero cannot be completely suppressed, even for small intensities, and thus causes background events for the lifetime determination. In order to understand how the ICD lifetime is contained in the time-dependent trace of Fig. 5, we have performed classical simulations, which are discussed in the following Sec. 3.2.
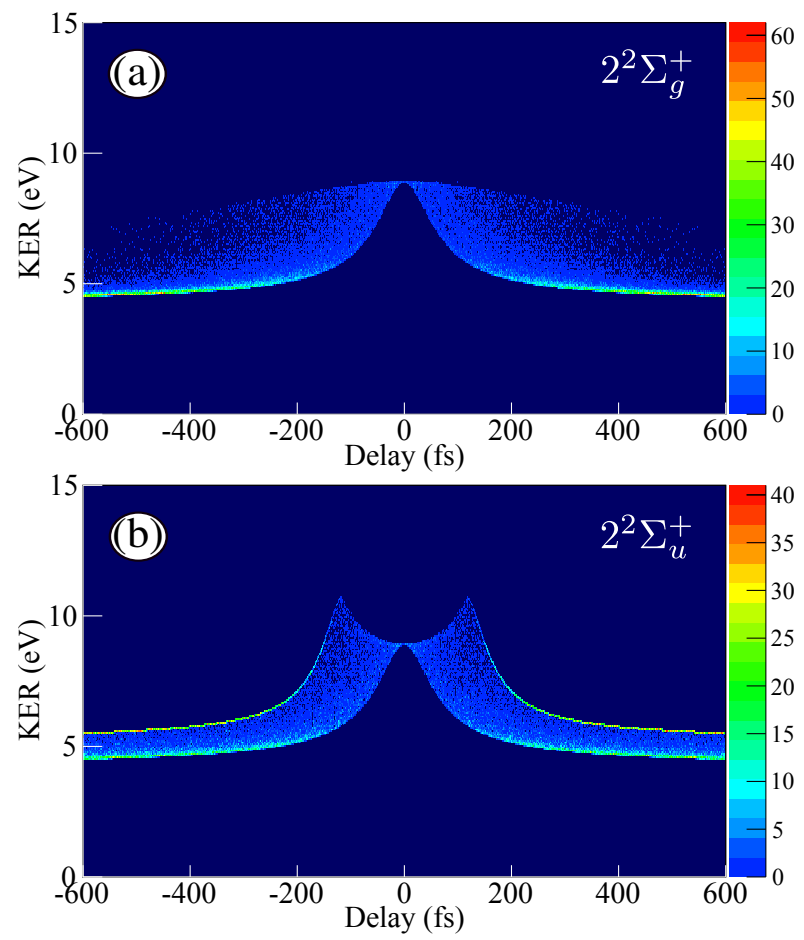

Figure 6: Classical simulation of the delay-dependent KER spectrum for $\mathrm{Ne}^{+}-\mathrm{Ne}^{2+}$ ion pairs produced after ICD. (a) Population of the flat intermediate $2^{2} \Sigma_{g}^{+}$curve. (b) Population of the attractive intermediate $2^{2} \Sigma_{u}^{+}$curve.

\subsection{Classical Simulations}

The classical simulation used to model our pump-probe spectra treats molecular motion as a point-like particle moving on PECs according to Newton's classical equation of motion. The respective PECs for $\mathrm{Ne}_{2}$ have been calculated [36] and can therefore be used as input for the simulation. The simulation starts by placing the point-like classical particle either on the $2^{2} \Sigma_{g}^{+}$or $2^{2} \Sigma_{u}^{+}$curve at $R_{\text {eq }}=3.1 \AA$, which models single-photon absorption from the dimer ground state. Both states are assumed to be populated with equal probability, which is in line with experimental observations [37]. While the particle moves towards smaller $R$ when placed on the attractive $2^{2} \Sigma_{u}^{+}$curve, it will more or less stay at rest on the virtually flat $2^{2} \Sigma_{g}^{+}$PEC. The ICD of the intermediate state is implemented by assuming a random decay time $t$ that follows the exponential propability distribution $\exp ^{-t / \tau}$ with the ICD lifetime $\tau$. At $t$ the assigned lifetime the system jumps onto the repulsive $\propto 1 / R$ curve where it pursues its motion until the probe pulse arrives after an adjustable time delay $t_{d}$, thereby promoting the system onto the steeper $\propto 2 / R$ PEC.

In Fig. 6, the comparisons of the classical simulations for $2^{2} \Sigma_{g}^{+}$and $2^{2} \Sigma_{u}^{+}$are shown with an exemplary ICD lifetime of $\tau=100 \mathrm{fs}$. If the $2^{2} \Sigma_{g}^{+}$PEC is populated, the system will remain close to $R_{\text {eq }}$. Thus, the shape of the spectrum is determined by the interplay of the exponential decay and the arrival time of the probe pulse. The time when ICD occurs is simulated by an exponential probability distribution, thus it may take place at any time after the creation of the $2 s$ vacancy. If 
ICD occurs immediately after the pump pulse, the repulsive $1 / R$ curve is populated early giving the nuclei the largest possible time to dissociate before the probe pulse further ionizes the system into $\mathrm{Ne}^{+}-\mathrm{Ne}^{2+}$ fragments. Thus, events with a small decay time are located close to the low-energy cut-off of Fig. 6 because the steep $\mathrm{Ne}^{+}-\mathrm{Ne}^{2+}$ curve is populated at large internuclear distances where the Coulomb-potential-energy gain is already small. In contrast, for dimers decaying just before the probe pulse arrives, the internuclear distance when the fragments are further ionized is still close to $R_{\text {eq }}$ resulting in a much larger energy gain on the steep $\mathrm{Ne}^{+}-\mathrm{Ne}^{2+}$ PECs. Thus, these events occur at larger KERs.

In Fig. 6(b), the corresponding spectrum for the population of the intermediate $2^{2} \Sigma_{u}^{+}$state is shown. It features an additional wing-like structure compared to the $2^{2} \Sigma_{g}^{+}$state, which is due to nuclear motion before ICD has occurred. The lowenergy cut-offs in Fig. 6(a) and (b) are identical because they have the same origin: For a fast ICD, nuclear motion on the intermediate $2^{2} \Sigma_{u}^{+}$curve is negligible, resulting in the well-known behavior of delayed ionization. Instead, if ICD takes place late, the classical particle will start to move towards smaller $R$ leading to a smaller $R$ by the time the dimer ion decays. Therefore, the steepest PEC, $\mathrm{Ne}^{+}-\mathrm{Ne}^{2+}$, is accessed at internuclear distances smaller than $R_{\text {eq }}$ resulting in KERs higher than those expected at $R_{\text {eq }}$. However, the KER in Fig. 6(b) rises only up to roughly $11 \mathrm{eV}$, which translates into an internuclear distance of $R=2.6 \AA$. ICD is only energetically allowed as long as the $2^{2} \Sigma_{u}^{+}$curve lies above the $\mathrm{Ne}^{+}-\mathrm{Ne}^{+}$curve and the crossing region of the two curves lies around $R=2.6 \AA$. Thus, no KERs higher than the one corresponding to a Coulomb explosion at the crossing point occurs.

In the simulation, a small number of $\mathrm{Ne}_{2}^{+}$ions reach this crossing point and the question arises how to deal with them. In Fig. 6(b), we have assumed immediate decay at the crossing point onto the $\mathrm{Ne}^{+}-\mathrm{Ne}^{+}$curve, which causes the sharp cut-off at high energies beyond $120 \mathrm{fs}$. Alternatively, we have also performed simulations where the classical particle may cross the intersection. In this region, dimers can only decay radiatively, which is not included in our simulation. Therefore, those dimers that have crossed the intersection will eventually return to the region of internuclear distances where ICD is allowed. This difficulty demonstrates the limitations of our model of classical point-like particles moving on PECs. Furthermore, for quantum-mechanical calculations employing nuclear wavepacket propagation the wave packet exhibits a certain width. The fraction of the wave packet beyond the crossing may decay only radiatively because the ICD decay width drops to zero. However, even if the wave packet propagates to smaller $R$ the decay rate does not vanish completely as some part of the wave function remains before the crossing. This condition is fulfilled for $\mathrm{Ne}_{2}^{+}\left(2 s^{-1}\right)$ for all times, as the highest vibrational levels are predominantly populated by the pump pulse and these levels exhibit nuclear wave functions that are extended to larger $R$. Therefore, the probability density before the crossing never vanishes completely and ICD remains allowed at all times.

Experimentally, we are not able to distinguish between the $2^{2} \Sigma_{u}^{+}$and the $2^{2} \Sigma_{g}^{+}$state. Fig. 8 shows the superposition of

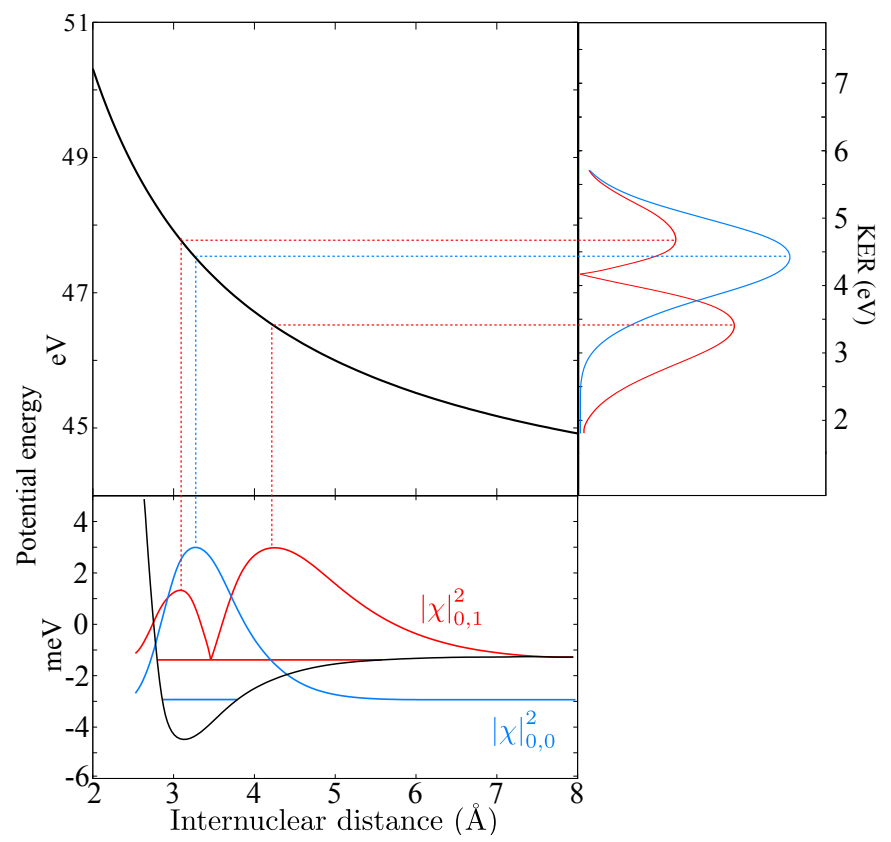

Figure 7: Mapping of the squared nuclear wave function via projection onto the $1 / R$ PEC. The squared nuclear wave functions $|\chi|_{0,0}^{2}$ of the lowest (in blue) and $|\chi|_{0,1}^{2}$ of the first excited (in red) vibrational level are mapped onto the $\mathrm{Ne}^{+}-\mathrm{Ne}^{+}$Coulomb curve and translated into the corresponding KER distribution. Here, instantaneous population of the Coulomb curve is assumed. However, nuclear motion in the intermediate state will affect the shape of the wave packet. Thus, comparing the shape of the measured KER distribution and that expected from the ground state wave function reveals important information on the nuclear dynamics in the intermediate state. Wave functions are taken from Ref. [38].

the simulation for equal contribution of each intermediate state. The general behavior of the time-dependent experimental trace (cf. Fig. 5) is well reproduced by our simulation supporting the proposed pathway. However, the characteristic increases of the KER due to nuclear motion on the $2^{2} \Sigma_{u}^{+}$are not observed in the data. The absence of this feature has various reasons: Counts from direct multi-photon absorption overlap energetically with the expected KER increase and cannot be separated from the pump-probe contribution with statistical significance. In addition, our simulation was performed at a fixed initial internuclear distance $R_{\mathrm{eq}}$, which is in contrast to the spatial distribution of the ground-state wave function, as shown in Fig. 7. If we perform the simulation with a distribution of initial internuclear distances according to the ground state wave function the winglike structure gets washed out, as demonstrated in Fig. 8(b).

Up to now, we have not discussed the observation that the simulated rate of $\mathrm{Ne}^{+}-\mathrm{Ne}^{2+}$ ion pairs decreases for small pump-probe delay, which is especially apparent in Fig. 8. In order to more closely investigate this time-dependence we have to evaluate the projection of all KERs onto the delay axis, as shown in the lower panel of Fig. 8(b). The $\mathrm{Ne}^{+}-\mathrm{Ne}^{2+}$ ion yield vanishes for small delays and saturates for larger delays. 

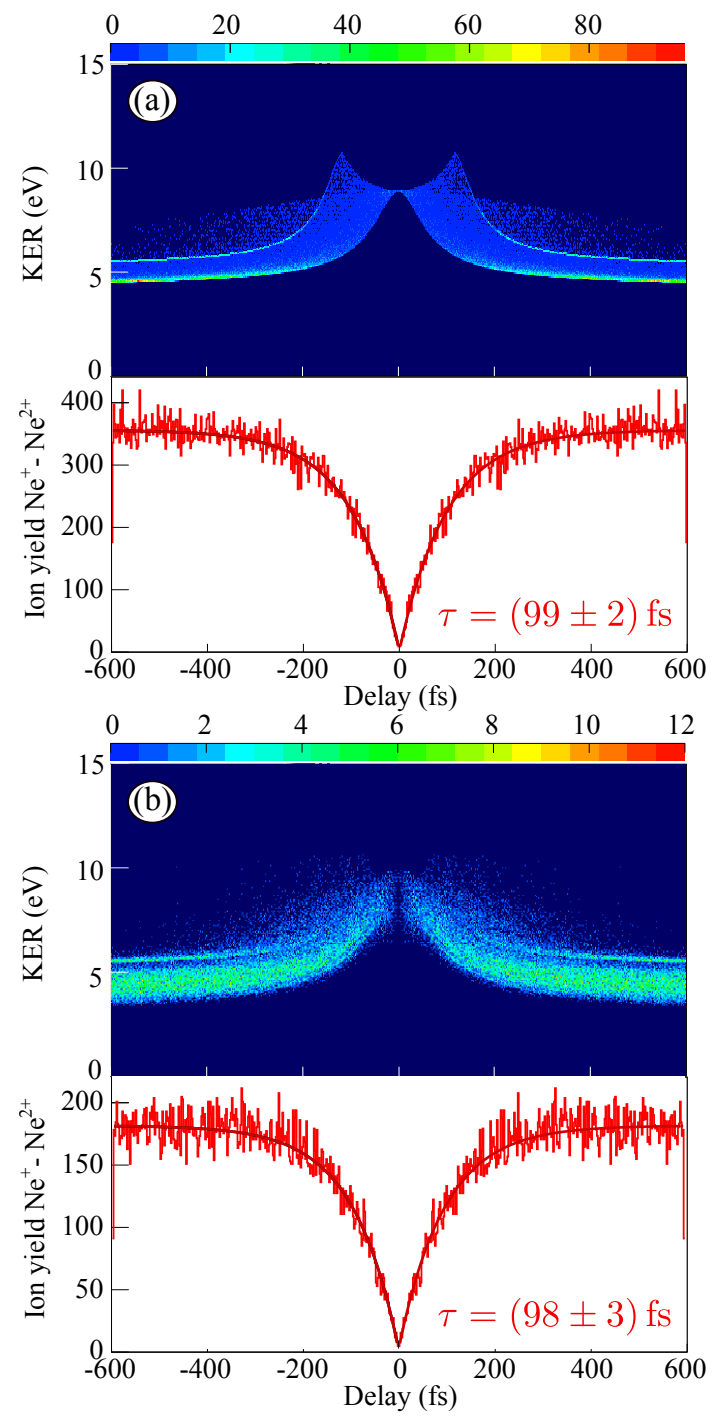

Figure 8: Simulation of the delay-dependent KER spectra of coincident $\mathrm{Ne}^{+}-\mathrm{Ne}^{2+}$ ion pairs considering both intermediate states $2^{2} \Sigma_{u}^{+}$and the $2^{2} \Sigma_{g}^{+}$excited together (Fig. 6(a)/(b) superposed) for an ICD lifetime of $100 \mathrm{fs}$. The projection of all KERs onto the delay axis is strongly time dependent and the dependence of the ion yield returns the input lifetime of the simulation. (a) Simulation for an initial fixed internuclear distance of $R_{\text {eq }}=3.1 \AA$. (b) Simulation with $R$ distributed according to the squared vibrational ground state wave function $|\chi|_{0,0}^{2}$, shown in Fig. 7.

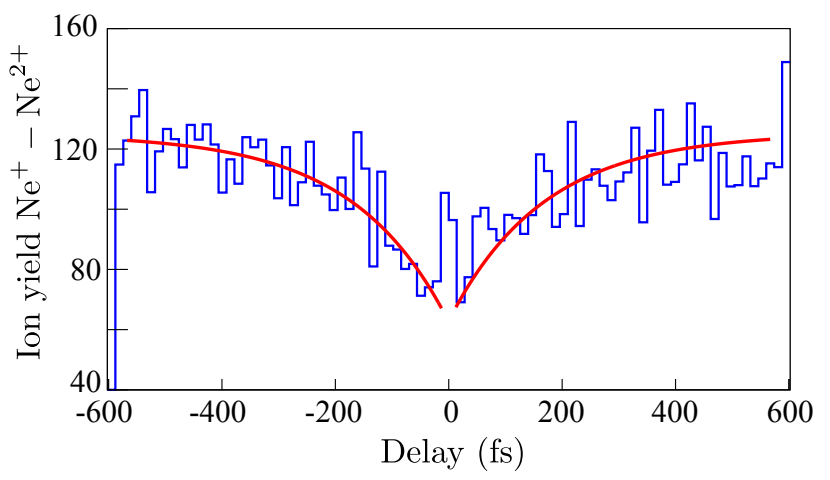

Figure 9: Projection of all KERs in the time-dependent $\mathrm{Ne}^{+}-$ $\mathrm{Ne}^{2+}$ spectrum onto the delay axis. The red lines indicate the increasing ion yield from small towards larger delays.

This behavior becomes clear when recalling the involved PECs: Coincident $\mathrm{Ne}^{+}-\mathrm{Ne}^{2+}$ ion pairs are only produced when ICD has already occurred by the time the probe pulse impinges. For small delays, only few dimers have undergone ICD and consequently only a small fraction of $\mathrm{Ne}^{+}-\mathrm{Ne}^{+}$ion pairs are further ionized to $\mathrm{Ne}^{+}-\mathrm{Ne}^{2+}$. By contrast, for large delays almost all $\mathrm{Ne}_{2}^{+}\left(2 s^{-1}\right)$ ions have decayed into $\mathrm{Ne}^{+}-\mathrm{Ne}^{+}$pairs and thus the number of $\mathrm{Ne}^{+}-\mathrm{Ne}^{2+}$ ions is large as well. Performing an exponential fit to the time-dependent ion yield returns the input lifetime of the simulation. Thus, by analyzing the $\mathrm{Ne}^{+}-\mathrm{Ne}^{2+}$ ion yield we have found a tool to directly measure the ICD lifetime.

\subsection{Lifetime Determination}

In order to determine the ICD lifetime from our experimental data, we proceed the same way as introduced for the simulation in Sec. 3.2: The measured time-dependent KER spectrum of coincident $\mathrm{Ne}^{+}-\mathrm{Ne}^{2+}$ ions is projected onto the delay axis, as shown in Fig. 9. The projection exhibits a clear drop in the ion yield towards small delays as predicted by the simulation. The origin of the constant offset in the ion yield in Fig. 9 will be discussed in detail in Sec. 3.4. To avoid fluctuations in the ion yield due to imperfections of the delay stage, the projected spectra are normalized to the total time the delay stage spent at each scanning step.

In order to make full use of the collected data, the counts for negative delays were added to those of the respective positive delays (cf. Fig. 10(a)) as the experiment is mirror-symmetric with respect to time delay zero. However this assumption is only true, if the FEL intensity was distributed equally among the split beams and spatial overlap was kept constant for all delays. We inspected the beams' distribution and overlap before each delay scan and confirmed constant conditions by comparing the ion yield of the constant and the time-dependent trace in Fig. 5(a) for positive and negative delays. Fig. 10(b) shows the projection of all coincident $\mathrm{Ne}^{+}-\mathrm{Ne}^{2+}$ ion pairs in the energy range from $0 \mathrm{eV}-$ alsoload $11 \mathrm{eV}$ onto the delay axis. An exponential fit yields an ICD lifetime of $150 \mathrm{fs} \pm 50 \mathrm{fs}$ with a purely statistical uncertainty. An upper limit of the temporal 

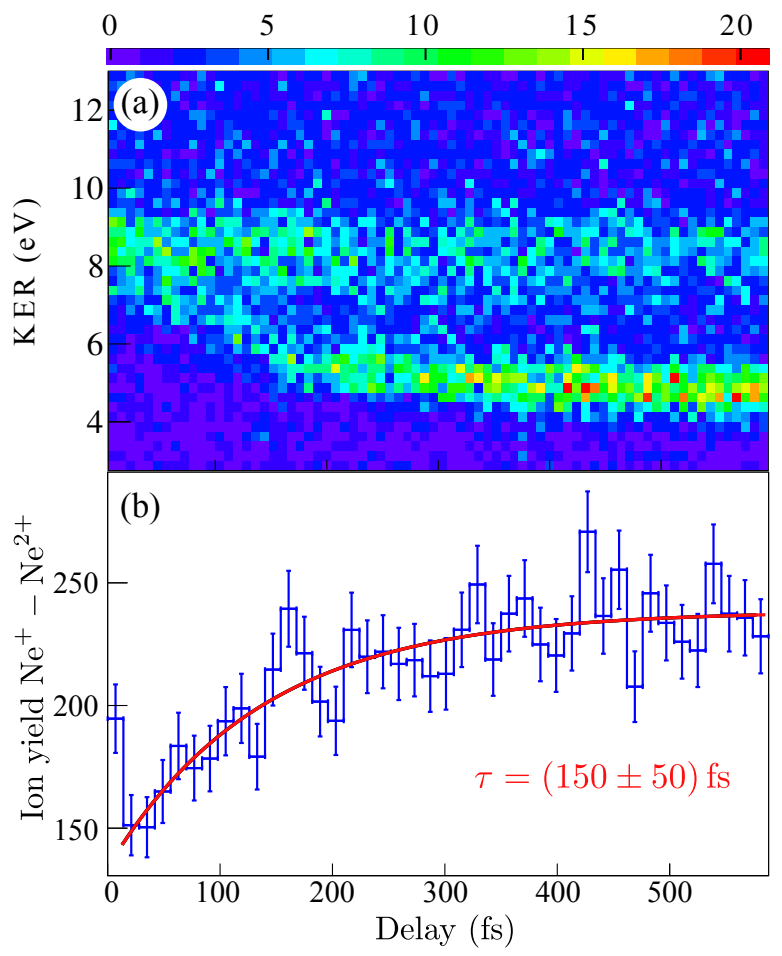

Figure 10: (a) Delay-dependent KER spectrum of coincident $\mathrm{Ne}^{+}-\mathrm{Ne}^{2+}$ ions where negative delays from Fig. 5(a) are flipped onto the positive delay range in order to improve statistics. (b) Projection of KERs between 0 and $11 \mathrm{eV}$ onto the delay axis. The exponential fit (in red) returns an ICD lifetime of $150 \mathrm{fs} \pm 50 \mathrm{fs}$.

resolution is determined by the uncertainty arising from the finite pulse length: For a Gaussian pulse with $\sigma=26$ fs the uncertainty due to the temporal overlap of pump and probe pulse amounts to $\pm \sigma \cdot \sqrt{2}= \pm 36$ fs. Thus, the accuracy of the lifetime determination is not yet limited by the pulse duration. Moreover, we expect an even better temporal resolution due to the spiky structure of the FEL pulses [39]. The given pulse length of $60 \mathrm{fs}$ (FWHM) is averaged over a larger number of pulses while each individual pulse exhibits spikes with a width of a few fs, corresponding to the temporal coherence length [40]. The peak structure manifests in our data as a sharp coherence peak at time delay zero. As it is unrelated to ICD, it has to be excluded for the lifetime fit. Still it influences the fit result to larger lifetimes and consequently our result should be understood as an upper limit for the lifetime. On the other hand, the peak allows us to determine the zero of the time-delay axis, when the pulses have perfect temporal overlap, to an accuracy of a few fs. This is crucial for adding the ion yield of negative delays onto the positive ones.

\subsection{Competing Pathways}

In order to ensure that the increasing ion yield of $\mathrm{Ne}^{+}-\mathrm{Ne}^{2+}$ ion pairs is caused by ICD, we have to investigate all competing pathways leading to the same charge state very carefully. This may be done by identifying the relevant pathways and performing classical simulations to study their influence on the over-

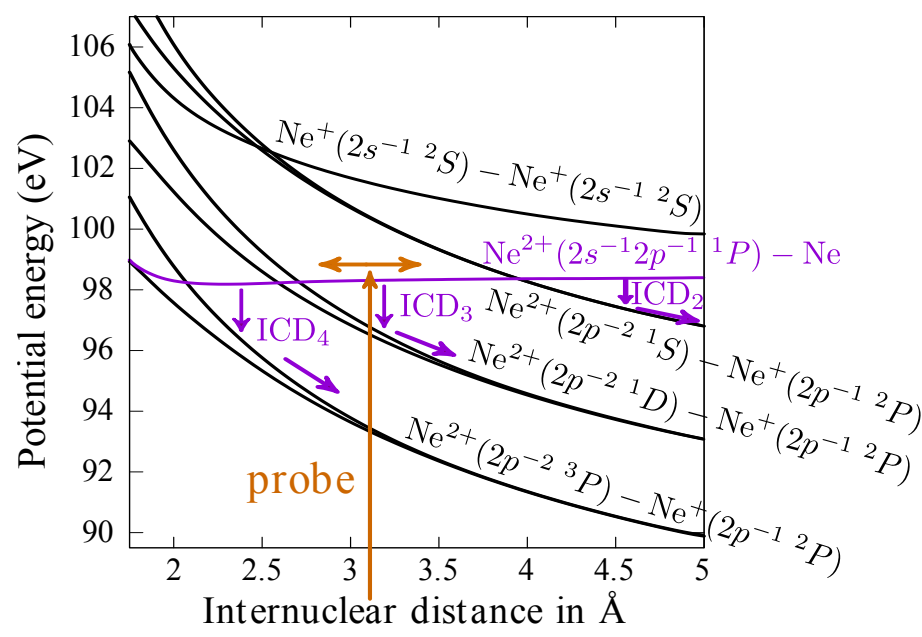

Figure 11: Competing pathways via the population of the $\mathrm{Ne}^{2+}\left(2 s^{-1} 2 p^{-1}{ }^{1} P\right)-\mathrm{Ne}$ potential curve (in purple). The indicated state can decay into background coincident $\mathrm{Ne}^{+}-\mathrm{Ne}^{2+}$ ion pairs via $\mathrm{ICD}_{2}, \mathrm{ICD}_{3}$ or $\mathrm{ICD}_{4}$. The orange arrow indicates $R_{\text {eq. }}$. The PECs are taken from [36].

all $\mathrm{Ne}^{+}-\mathrm{Ne}^{2+}$ ion yield. As the time-independent contribution from multi-photon absorption causes only a constant offset in the ion-yield spectrum the lifetime determination is not disturbed by this competing pathway. Relevant are only pathways with delay-dependent ion yield because those could influence the outcome of the lifetime fit. If the inner-valence-ionized dimer $\mathrm{Ne}_{2}^{+}\left(2 s^{-1}\right)$ absorbs another photon before ICD has occurred it will result in the fragmentation channels $\mathrm{Ne}^{+}-\mathrm{Ne}^{+}$ or $\mathrm{Ne}-\mathrm{Ne}^{2+}$. One particular state, $\mathrm{Ne}-\mathrm{Ne}^{2+}\left(2 s^{-1} 2 p^{-1}{ }^{1} P\right)$, also decays via ICD into $\mathrm{Ne}^{+}-\mathrm{Ne}^{2+}$ ion pairs and thus causes a time-dependent background. Fig. 11 shows a zoom into the PEC spectrum into the region from $90 \mathrm{eV}$ to $106 \mathrm{eV}$ illustrating the competing decay channels $\mathrm{ICD}_{2}, \mathrm{ICD}_{3}$, and $\mathrm{ICD}_{4}$ from the $\mathrm{Ne}-\mathrm{Ne}^{2+}\left(2 s^{-1} 2 p^{-1}{ }^{1} P\right)$ state. These ICD processes were predicted theoretically $[36,41]$ and have been confirmed experimentally [42]. However, in these studies the population of the decaying state was initiated by the creation of a $1 s$ core hole that decayed via an Auger cascade.

Here, the population of the $\mathrm{Ne}-\mathrm{Ne}^{2+}\left(2 s^{-1} 2 p^{-1}{ }^{1} P\right)$ state is either achieved by sequential two-photon absorption within one pulse or by a single-photon pump single-photon probe scheme for $\mathrm{Ne}_{2}^{+}\left(2 s^{-1}\right)$ ions that have not yet undergone ICD (cf. Fig.4). The latter pathway is most prominent for small time delays because shortly after the pump pulse only few dimers have undergone ICD and are consequently probed into the $\mathrm{Ne}-\mathrm{Ne}^{2+}\left(2 s^{-1} 2 p^{-1}{ }^{1} P\right)$ state. For small delays, the nuclei are given little time for motion in the intermediate $2^{2} \Sigma_{g}^{+}$ and $2^{2} \Sigma_{u}^{+}$states. Thus, the shape of the wave packet accessing the $\mathrm{Ne}-\mathrm{Ne}^{2+}\left(2 s^{-1} 2 p^{-1}{ }^{1} P\right)$ curve should still be close to that of the ground state and peak around $R_{\mathrm{eq}}$. The same argument holds for the population of the curve via two-photon absorption within the pulse duration of $60 \mathrm{fs}$. However, in order to enable decay via $\mathrm{ICD}_{2}$ internuclear distances beyond the curve crossing of the initial and final state at $3.8 \AA$ must be populated (cf. 
Fig. 11). Although the wave packet is broad, only a very small fraction of the distribution will exceed the crossing allowing the decay. Thus, the overall contribution of $\mathrm{ICD}_{2}$ is negligible.

$$
\begin{gathered}
\mathrm{Ne}-\mathrm{Ne}^{2+}\left(2 s^{-1} 2 p^{-11} P\right) \\
\downarrow \mathrm{ICD}_{2} \\
\mathrm{Ne}^{2+}\left(2 p^{-2}{ }^{1} S\right)-\mathrm{Ne}^{+}\left(2 p^{-12} P\right) .
\end{gathered}
$$

$\mathrm{ICD}_{3}$ instead takes place for internuclear distances larger than $\approx 2.6 \AA$ which covers the distance range at which most of the nuclear wave packet is launched. The decay occurs via direct ICD:

$$
\begin{gathered}
\mathrm{Ne}-\mathrm{Ne}^{2+}\left(2 s^{-1} 2 p^{-11} P\right) \\
\downarrow \mathrm{ICD}_{3} \\
\mathrm{Ne}^{2+}\left(2 p^{-2}{ }^{1} D\right)-\mathrm{Ne}^{+}\left(2 p^{-12} P\right) .
\end{gathered}
$$

The transition time is predicted to be $80 \mathrm{fs}$ [41]. For a fast decay, only little nuclear motion takes place resulting in a KER distribution of the $\mathrm{Ne}^{+}-\mathrm{Ne}^{2+}$ fragments corresponding to distances around $R_{\text {eq }}$. Therefore, events from $\mathrm{ICD}_{3}$ are expected to emerge at similar KERs as those from direct multi-photon absorption and will consequently be indistinguishable from them. The maximal cut-off energy for this decay channel is computed by translating the internuclear distance of the curve crossing at 2.6 $\AA$ into the corresponding KER of $11 \mathrm{eV}$.

The transition $\mathrm{ICD}_{4}$ in Fig. 11 takes place for $R$ as small as $2.13 \AA$ resulting in a maximum KER of $13.5 \mathrm{eV}$. However, in our estimate of purely Coulombic PECs might not be adequate anymore for these small distances. It is important to note that $\mathrm{ICD}_{4}$ differs from the ICD transitions discussed so far. It is not mediated by exchange of a virtual photon alone. Instead an additional spin-flip is needed, as a singlet $P$-state has to be transformed into a triplet $P$-state. However, a transition including a spin-flip is dipole-forbidden and thus strongly suppressed [36]. Therefore, different types of ICD occur: exchange ICD (exICD) or ETMD. As shown in Fig. 13, both require the exchange of electrons, contrasting the "normal" ICD where energy is transferred via a virtual photon. The decay reads

$$
\begin{gathered}
\mathrm{Ne}-\mathrm{Ne}^{2+}\left(2 s^{-1} 2 p^{-11} P\right) \\
\downarrow \text { exICD + ETMD } \\
\mathrm{Ne}^{2+}\left(2 p^{-23} P\right)-\mathrm{Ne}^{+}\left(2 p^{-12} P\right) .
\end{gathered}
$$

In exICD, the inner-valence vacancy located at the $\mathrm{Ne}^{2+}$ ion is filled by a $2 p$ electron from the neutral $\mathrm{Ne}$ neighbor, thereby removing another $2 p$ electron from the formerly excited ion resulting in coincident $\mathrm{Ne}^{+}-\mathrm{Ne}^{2+}$ ions. ETMD is different in the sense, that the excess energy after population of the innervalence hole is transferred to a $2 p$ electron from the formerly neutral neighbor atom, which is then ionized. Both processes are illustrated in Fig. 13. As mechanisms involving the migration of electrons require sufficient orbital overlap, exICD and

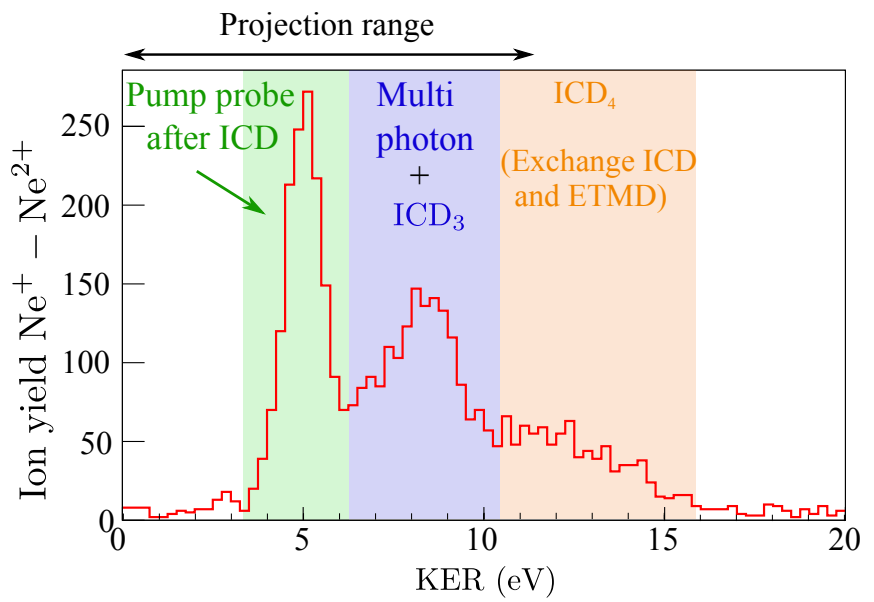

Figure 12: KER spectrum of coincident $\mathrm{Ne}^{+}-\mathrm{Ne}^{2+}$ ions at large delays. The green shaded area marked with "Pump probe after ICD" contains the counts that are relevant for the lifetime determination. Note that the pump-probe region is extended up to $11 \mathrm{eV}$, when considering all delays. Therefore, it will energetically overlap with the blue shaded "Multi photon $+\mathrm{ICD}_{3}$ " region. The projection range used for the lifetime determination in Fig. 10 is indicated on the top $\mathrm{X}$-axis.

ETMD can only occur at smaller $R$ compared to "normal" ICD and thus demand nuclear motion prior to the decay. For smaller distances, the decay rates can be strongly enhanced due to the orbital overlap [43]. However, nuclear motion takes time and causes the electron-transfer processes to be much slower than ICD via virtual-photon exchange [11].

Fig. 11 shows an overview of the different processes leading to coincident $\mathrm{Ne}^{+}-\mathrm{Ne}^{2+}$ ions and the corresponding relevant energy ranges are indicated in Fig. 12. The high-energetic contribution in the range from $11 \mathrm{eV}$ to $16 \mathrm{eV}$ stems most likely from the previously discussed exICD and ETMD processes (indicated as $\mathrm{ICD}_{4}$ in Fig. 11). The peak around $5 \mathrm{eV}$ stems from the discussed asymptotic limit of our desired pump-probe channel. For small time delays, it creates a signal in the intermediate KER range of $6 \mathrm{eV}$ to $11 \mathrm{eV}$ where it overlaps with the contributions from sequential multi-photon absorption and from $\mathrm{ICD}_{3}$.

\subsection{Simulation of Competing Processes}

The delay-dependent KER trace that contains the ICD lifetime cannot overcome an energy of $\approx 11 \mathrm{eV}$ due to the curve crossing at $2.6 \AA$. At this distance, the $2^{2} \Sigma_{g, u}^{+}$curves intersect the repulsive $\mathrm{Ne}^{+}-\mathrm{Ne}^{+}$curves and for smaller $R$, ICD is energetically not possible anymore. Therefore, the competing processes occurring via exICD and ETMD, which arise in the KER spectrum at higher values than $\approx 11 \mathrm{eV}$, are suppressed by projecting only smaller KERs than that onto the delay axis. All time-independent processes will cause a constant offset for the ion yield but do not disturb the overall shape of the decay curve. The only remaining delay-dependent background stems from $\mathrm{Ne}_{2}^{+}\left(2 s^{-1}\right)$ ions that are further ionized by the probe pulse to the $\mathrm{Ne}-\mathrm{Ne}^{2+}\left(2 s^{-1} 2 p^{-1}{ }^{1} P\right)$ state and decay via $\mathrm{ICD}_{3}$. Thus, 

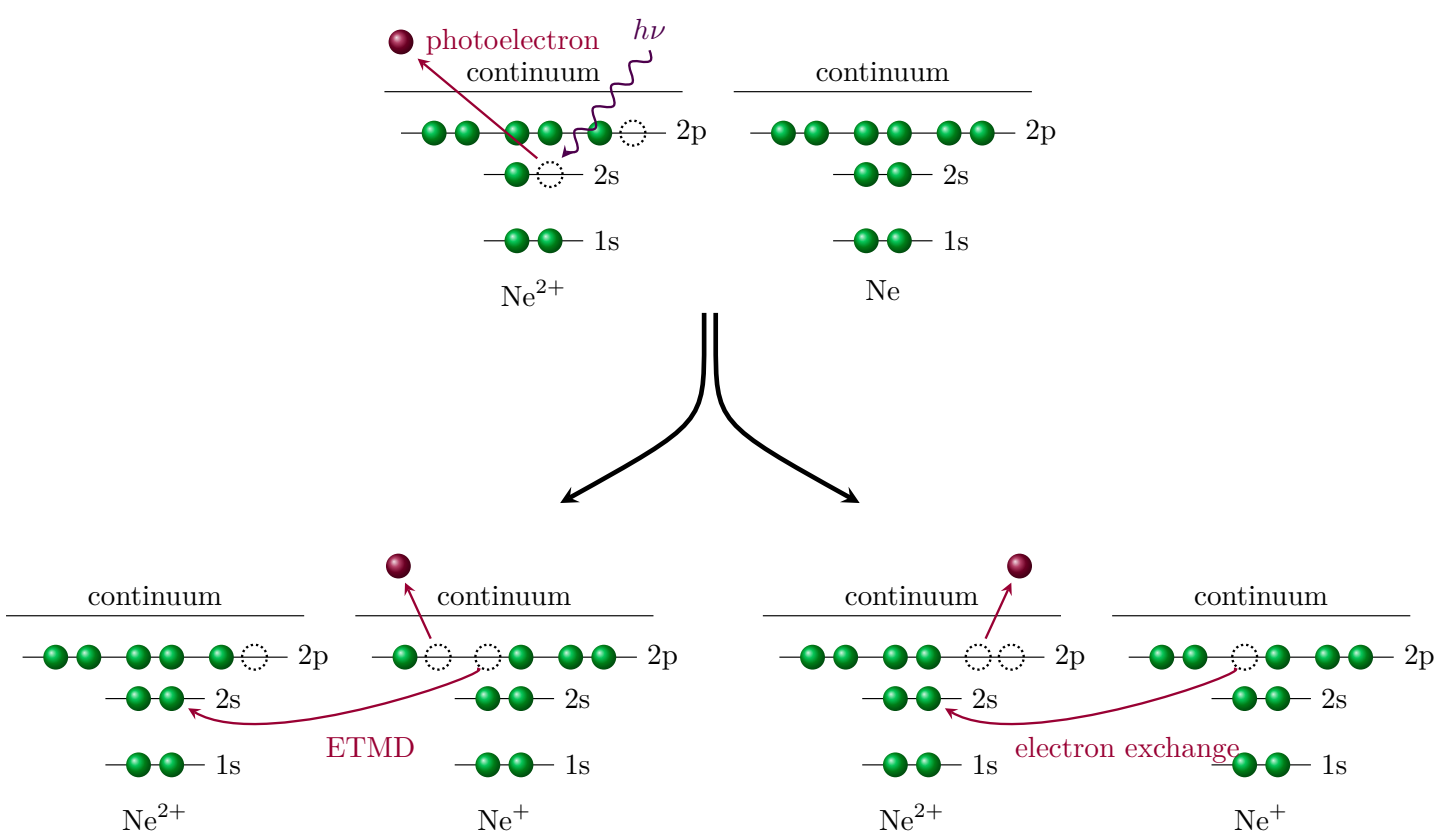

Figure 13: Comparison of exICD (right) and ETMD (left) for $\mathrm{ICD}_{4}$. In exICD, the relaxation energy is transferred to an electron of the initially inner-valence ionized side, while in ETMD the energy is transferred to the initially neutral side.

we will further investigate the influence of this pathway on the lifetime fit by utilizing our classical pump-probe simulation.

The simulation starts by populating the intermediate $2^{2} \Sigma_{u}^{+}$or $2^{2} \Sigma_{g}^{+}$potential curve and classical propagation in time until ICD occurs or the probe pulse arrives. Only if the latter event takes place first, the particle is placed on the $\mathrm{Ne}-\mathrm{Ne}^{2+}\left(2 s^{-1} 2 p^{-11} P\right)$ curve from where it decays onto a Coulombic $\mathrm{Ne}^{+}-\mathrm{Ne}^{2+}$ curve. The lifetime of $\mathrm{ICD}_{3}$ does not alter the delay-dependence of the $\mathrm{Ne}^{+}-\mathrm{Ne}^{2+}$ ion yield as long as the lifetime is much shorter than radiative decay. This condition is well met and thus all dimers will decay via $\mathrm{ICD}_{3}$. Therefore, the population of the yield of coincident $\mathrm{Ne}^{+}-\mathrm{Ne}^{2+}$ ions produced via the competing pathway is expected to be large for small delays and decreasing towards larger delays, which is inverse to the behavior of the primary ICD process.

Fig. 14 shows the result of a simulation of the competing process with an exemplary input lifetime of $100 \mathrm{fs}$, starting from the equilibrium internuclear distance $R_{\text {eq }}$. The timeindependent feature at $9 \mathrm{eV}$ corresponds to the pathway via the intermediate state $2^{2} \Sigma_{g}^{+}$. It is constant because nuclear motion in the intermediate state is negligible and the decay of the doubly ionized dimer is assumed to be instantaneous. The $\mathrm{ICD}_{3}$ time dependence is irrelevant for the ion yield as discussed earlier. Introducing the finite lifetime of $\mathrm{ICD}_{3}$ into the simulation would yield a spread along the KER axis but it would not influence the delay-dependence. The time-dependent trace in the upper panel of Fig. 14 corresponds to the population of the intermediate $2^{2} \Sigma_{u}^{+}$state. The nuclear motion causes the characteristic KER increase within the trace. As we probe the number of ions that still dwell in the intermediate $2 s$ hole state the projection of the ion yield produced via the competing process also returns the input lifetime and could in principle also be used for the lifetime determination.

The population of the $\mathrm{Ne}-\mathrm{Ne}^{2+}\left(2 s^{-1} 2 p^{-1} 1 P\right)$ PEC is only one possibility out of many options that can be reached after single-photon ionization of the intermediate $\mathrm{Ne}_{2}^{+}\left(2 s^{-1}\right)$ ion. Thus, in order to evaluate the relevance of the competing process we will count the total number of accessible electronic states and compare it with the direct ICD pathway. Fig. 4 shows only a small selection of accessible curves. A complete overview may be found in Ref [36]. In Tab. 1, the four possible electronic states are listed and weighted according to their spin multiplicity. Creating another $2 s$ vacancy is unlikely and thus left out of the following considerations. Including the spin multiplicity, the probability to access the $\mathrm{Ne}-\mathrm{Ne}^{2+}\left(2 s^{-1} 2 p^{-1}{ }^{1} P\right)$ state is only $1 / 6$ for a photon that is absorbed by the $\mathrm{Ne}_{2}^{+}\left(2 s^{-1}\right)$ ion. Note that this is a pessimistic estimate as the third process in Tab. 1 should be more probable because a neutral Ne is ionized and ETMD and exICD, which reduce the rate of $\mathrm{ICD}_{3}$, are not taken into account.

In Fig. 15, a comparison between a simulation including the competing pathway and one without is shown with an exemplary input lifetime of $100 \mathrm{fs}$. The direct and the competing process are normalized in the following way: Each dimer that has undergone ICD will end up in a $\mathrm{Ne}^{+}-\mathrm{Ne}^{2+}$ state when further ionized by another photon from the probe. But only $1 / 6$ of dimers that have not yet decayed via ICD will result in $\mathrm{Ne}^{+}-\mathrm{Ne}^{2+}$ ion pairs. Therefore, the contribution of the competing process in the simulation occurs suppressed by a factor of 6 . The lifetime fit of the total ion yield is almost unaffected. Thus, $\mathrm{ICD}_{3}$ can be assumed to not affect the lifetime determination. 


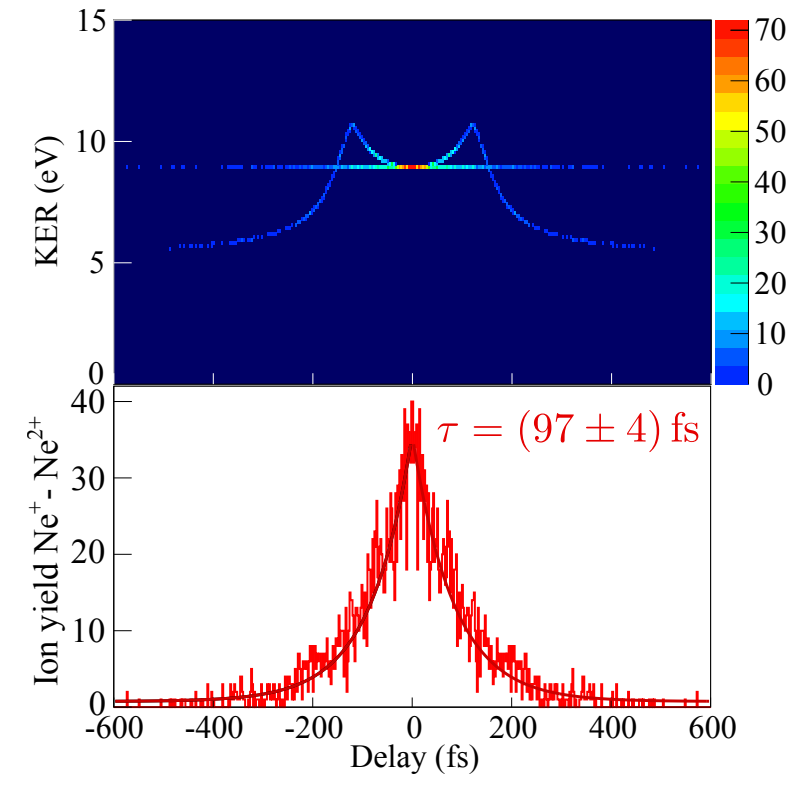

Figure 14: Simulation of the competing process $\mathrm{ICD}_{3}$ with an input lifetime of $100 \mathrm{fs}$. The upper panel shows the delaydependent KER of $\mathrm{Ne}^{+}-\mathrm{Ne}^{2+}$ ions for both intermediate states and the lower panel shows the projection of all events onto the delay axis. An exponential fit (in red) also returns the ICD lifetime as the rate of competing ion pairs decreases at the rate the direct ICD rate increases.

\subsection{Comparison with Theory}

Due to the availability of numerous different calculations, $\mathrm{Ne}_{2}$ serves as an excellent prototype-system to benchmark ICD theory. Calculations that include nuclear motion are challenging because the electronic-decay widths vary strongly with the internuclear distance, as shown in Fig. 2. Since the decaywidths calculations for a single distance alone are already highly demanding, most theoretical studies focus on the decay rate at the equilibrium internuclear distance. Therefore, we will first discuss some purely electronic calculations, which neglect nuclear motion and treat ICD as if it happened at a fixed internuclear distance. This is necessary, as those build the foundation for studies including nuclear motion which will be discussed subsequently.

The purely electronic calculations were almost exclusively carried out for the ${ }^{2} \Sigma_{g}$ state at an internuclear distance of $R=$ $3.2 \AA$. Only the results that are expected to be most accurate are discussed here. An early calculation employing the ComplexAbsorbing-Potential (CAP) method with Multi-Reference Configuration Interaction (MRCI) expansion yielded a value of $\tau=$ $64 \mathrm{fs}$ [45]. A further study was carried out in the CAP framework using the Algebraic-Digrammatic-Construction (ADC) method, which yielded a value of $\tau=92 \mathrm{fs}$ [46]. Using the Fano-ADC method to determine the ICD lifetime resulted in $\tau=82 \mathrm{fs}$ [12]. The disagreement with the experimental result may be attributed to nuclear motion not been taken into account. However the large spread of the predicted lifetimes is worth noting.

\begin{tabular}{lcc}
\hline Configuration & $\begin{array}{c}\text { Spin } \\
\text { multiplicity }\end{array}$ & $\begin{array}{c}\text { Cross section } \\
\text { (Mbarn) }\end{array}$ \\
\hline $\mathrm{Ne}-\mathrm{Ne}^{2+}\left(2 s^{-1} 2 p^{-1}{ }^{1} P\right)$ & 1 & 7 \\
$\mathrm{Ne}-\mathrm{Ne}^{2+}\left(2 s^{-1} 2 p^{-13} P\right)$ & 3 & 7 \\
$\mathrm{Ne}^{+}\left(2 p^{-1}{ }^{2} P\right)-\mathrm{Ne}^{+}\left(2 s^{-1}{ }^{2} S\right)$ & 2 & 7 \\
$\mathrm{Ne}^{+}\left(2 s^{-1}{ }^{2} S\right)-\mathrm{Ne}^{+}\left(2 s^{-1}{ }^{2} S\right)$ & 2 & 0.5 \\
\hline
\end{tabular}

Table 1: Electronic states accessible via single-photon absorption by the $\mathrm{Ne}^{+}\left(2 s^{-1}\right)$ ion, the competing ICD active state is colored red. In addition the spin multiplicity of each state is given. The approximate cross sections for each ionization step are those for isolated atoms and ions [44].

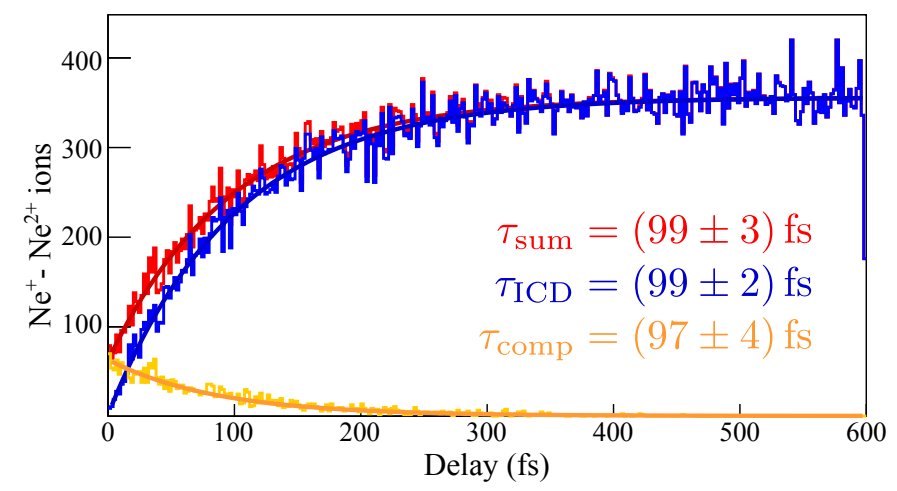

Figure 15: Simulation of the $\mathrm{Ne}^{+}-\mathrm{Ne}^{2+}$ ion yield for the direct ICD (blue), the competing pathway (orange) and the superposition of both (red). The results of an exponential fit for the respective spectra are also given.

In order to add nuclear motion to the calculations, nucleardynamics calculations must be carried out, i.e., time-dependent propagation of nuclear wave packets $[13,47]$ is used. In principle, this requires the knowledge of the decay rates for all internuclear distances. In practice, the $R$-dependent rates are determined by calculating static electronic decay rates for several discrete internuclear distances and interpolating in between, as shown in Fig. 2. The results of the three available calculations for $\Gamma$ including nuclear motion are shown in Fig. 16. All of them were performed starting from the vibrational ground state with the time-dependent propagation of wave packets. The respective method used for calculating the electronic-decay widths is also indicated in Fig. 16.

We begin by discussing the curve calculated via the CAP-CI method [2] in Fig. 16, which agrees the least with our measured lifetime. It was expected that the agreement of this calculation would be worse as it shows already a large deviation from the other lifetimes before nuclear motion was added. Therefore, it was not mentioned in the earlier discussion on purely electronic calculations. The curve of the CAP-MRCI method was obtained by inferring the $R$-dependence from the CAP-CI result by scaling it to the single data point obtained with the former. In contrast to the former, this method clearly underestimates the lifetime. The Fano-ADC result exhibits the best agreement, notably it lies within the error bars of our data. 


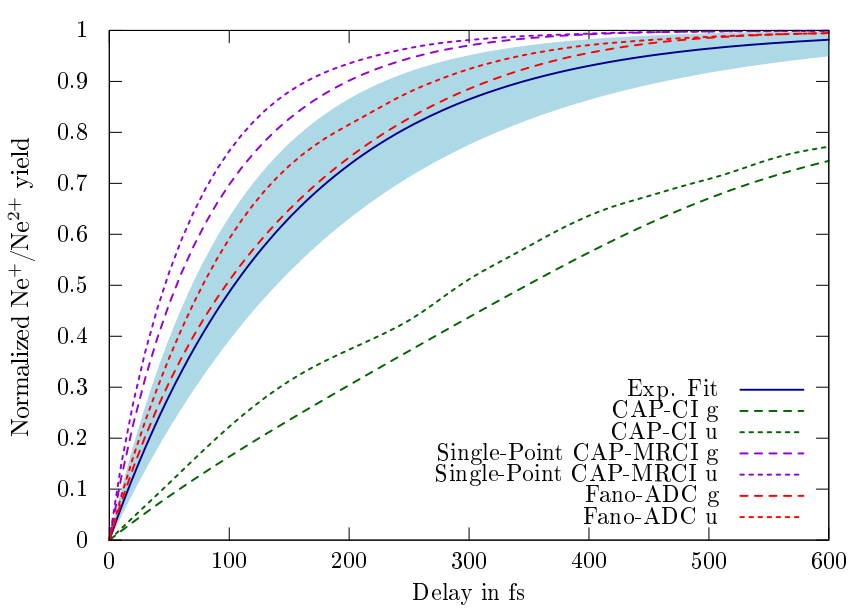

Figure 16: Comparison of experimental (exp.) and theoretical delay-dependent $\mathrm{Ne}^{+}-\mathrm{Ne}^{2+}$ ion yield: Calculations including nuclear motion start from the vibrational ground state and were performed with the time-dependent propagation of wave packets. The different methods for the determination of the electronic decay widths are color-coded in green (CAP-CI), red (Fano-ADC) and purple (CAP-MRCI). Both intermediate states are indicated, long dashed lines stand for the $2^{2} \Sigma_{g}^{+}$state and short dashed lines for the $2^{2} \Sigma_{u}^{+}$state. The experimental result is displayed in dark blue with their uncertainty as a light blue area.

Fig. 17 is analogue to Fig. 16 with the difference that the wave-packet calculations are performed starting from the first excited vibrational state. Obviously, the agreement is much worse and demonstrates nicely the strong dependence on the nuclear wave function of the initial state: The first excited state is extended towards larger $R$ where the decay width decreases strongly. Therefore, the calculations yield larger lifetimes compared to the ground-state wave function, which is centered at smaller $R$, as shown in Fig. 7.

\section{Discussion}

The ICD lifetime of $(150 \pm 50)$ fs for $\mathrm{Ne}_{2}^{+}\left(2 s^{-1}\right)$, discussed here, was determined under the assumption that the recorded ion yield can be approximated by an exponential fit. However, it was emphasized in Sec. 3.6 that this is only true for a dimer remaining at a fixed internuclear distance before ICD occurs. The decay width $\Gamma$ is thus different for all internuclear distances, as shown in Fig. 2. Nuclear motion will always be present when populating ICD active states via ionization, as done on our $\mathrm{Ne}_{2}$ experiment.. Therefore, we measure a decay time averaged over all internuclear distances that are covered from the creation of the inner-valence vacancy to the arrival of the probe pulse. This corresponds to the range of distances accessed by wave-packet dynamics in the intermediate state within $600 \mathrm{fs}$, the longest delay in the measurement. In addition, the intermediate state is populated with a spatial distribution according to the spatial width of the electronic ground state wave function.

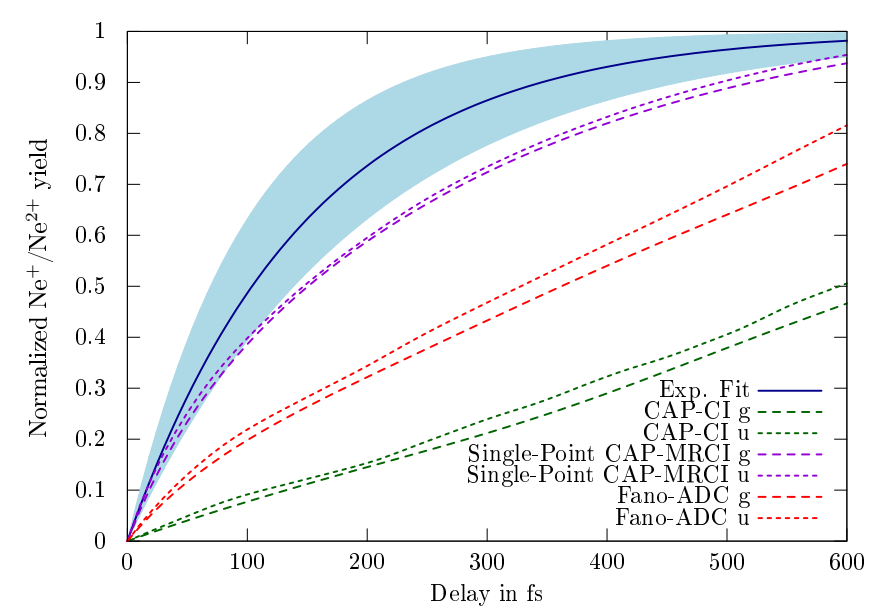

Figure 17: Same as Fig. 16, but with calculations starting from the vibrationally excited ground state.

The internuclear distance at which ICD occurred is imprinted in the KER. However, the KER does not carry information on how the dimer reached that particular distance and how long it took to get there. This would only be possible if all dimers started at $R_{\text {eq }}$ on a known intermediate PEC. However, in the experiment, we were not able to distinguish both intermediate states because the KERs overlapped energetically. For a measurement that detected also the ICD electrons the assignment to an intermediate state might be possible to some extend by considering the angular distribution of the emitted electrons [37]. In addition, the KER distribution is smeared out from the beginning due to the width of the ground-state wave function. Thus, we are neither able to determine the populated intermediate state nor the ground state internuclear distance. The situation is further complicated by experimental uncertainties, such as the finite pulse width, contributions from multi-photon absorption, limited statistics, and limited momentum resolution. Therefore, we have to average over both intermediate states and the dynamics taking place within the 600 fs of delay, which we do by assuming an exponential behavior.

The strongest deviation from an exponential behavior is expected for large delays because the assumption of an exponential decay gets worse the longer the wave packet evolves on the intermediate potential curves. This assumption is in line with the calculated curves in Fig. 16, which follow an exponential behavior for small to intermediate delays and deviate for large ones.

\section{Conclusion and Outlook}

We have exploited the unique properties of FEL radiation, i.e., short pulse duration and high intensities, to access ICD in $\mathrm{Ne}_{2}$ directly in the time domain. Employing an XUVpump-XUV-probe scheme we succeeded in determining the lifetime of the ICD-active $2 s$ innervalence vancancy in $\mathrm{Ne}_{2}^{+}$to $150 \mathrm{fs} \pm 50 \mathrm{fs}$. Our measurement agrees well with theory if nuclear motion prior to the decay is included in the calculations. 
From this, we infer the importance of nuclear motion for small systems, such as $\mathrm{Ne}_{2}$, undergoing ICD.

In order to ensure that the delay-dependent yield of $\mathrm{Ne}^{+}-$ $\mathrm{Ne}^{2+}$ ion pairs correctly reflects the ICD lifetime, we had to make sure that no other relaxation process is causing or influencing the observed behavior. This required the knowledge of the PEC spectrum. Since these spectra are available or can be calculated for various small systems, the presented pump-probe scheme will be applicable for other rare-gas and small molecular clusters. One limitation of the presented XUV-pump-XUVprobe scheme is the temporal resolution imposed by the duration of the XUV pulse itself. Therefore, fast ICDs on the few fs time-scale are not accessible in this manner, given the currently available pulse durations at FLASH. Another method to resolve the delayed emission of electrons is routinely applied in attosecond science for intrinsically synchronized XUV and IR pulses. This streaking method [48] has already been successfully employed to measure temporal shifts in photoemission [49]. At FLASH an analogous scheme can be applied using synchronized XUV- and THz-pulses. Here, electrons ejected by the XUV pulse are emitted into a THz-field and dependent on the time of emission they experience a characteristic momentum shift. Therefore, when compared to an instantaneously emitted electron, delayed electrons carry a characteristic momentum shift, which has the emission time imprinted.

In conclusion, the presented experiment on $\mathrm{Ne}_{2}$ shows how the properties of ultrashort intense XUV or X-ray radiation delivered by FELs can be used to enrich the field of ICD studies.

\section{Acknowledgments}

We acknowledge technical support from B. Knape and C. Kaiser, and thank the scientific and technical team at FLASH for optimal beam conditions. We acknowledge support by the DFG through the cluster of excellence: Munich Centre for Advanced Photonics (MAP). A.R. was supported by the Chemical Sciences, Geosciences, and Biosciences Division, Office of Basic Energy Sciences, Office of Science, U.S. Department of Energy. Y.H.J. is grateful for support from the National Basic Research Program of China (973 Program) (grant 2013CB922200), the NSFC (Grant NO. 11420101003 and 11274232), and the Shanghai Pujiang Program (grant 13PJ1407500). V.A. is supported by the Engineering and Physical Sciences Research Council (EPSRC, UK) through the Research Fellowship (award EP/H003657/1) and the Programme Grant on Attosecond Dynamics (award EP/I032517) as well as by the Deutsche Forschungsgemeinschaft through the Research Unit 1789.

\section{References}

[1] L. S. Cederbaum, J. Zobeley, F. Tarantelli, Giant Intermolecular Decay and Fragmentation of Clusters, Phys. Rev. Lett. 79 (1997) 4778.

[2] R. Santra, J. Zobeley, L. S. Cederbaum, N. Moiseyev, Interatomic Coulombic Decay in van der Waals Clusters and Impact of Nuclear Motion, Phys. Rev. Lett. 85 (2000) 4490.
[3] S. Marburger, O. Kugeler, U. Hergenhahn, T. Möller, Experimental Evidence for Interatomic Coulombic Decay in Ne Clusters, Phys. Rev. Lett. 90 (2003) 203401.

[4] N. V. Kryzhevoi, V. Averbukh, L. S. Cederbaum, High activity of helium droplets following ionization of systems inside those droplets, Phys. Rev. B 76 (2007) 094513.

[5] V. Averbukh, L. S. Cederbaum, Interatomic Electronic Decay in Endohedral Fullerenes, Phys. Rev. Lett. 96 (2006) 053401.

[6] G. Öhrwall, N. Ottosson, W. Pokapanich, S. Legendre, S. Svensson, O. Björneholm, Charge Dependence of Solvent-Mediated Intermolecular Coster-Kronig Decay Dynamics of Aqueous Ions, J. Phys. Chem. B 114 (2010) 17057-17061.

[7] B. Boudaïffa, P. Cloutier, D. Hunting, M. A. Huels, L. Sanche, Resonant Formation of DNA Strand Breaks by Low-Energy ( 3 to $20 \mathrm{eV}$ ) Electrons, Science 287 (2000) 1658-1660.

[8] E. Alizadeh, T. M. Orlando, L. Sanche, Biomolecular Damage Induced by Ionizing Radiation: The Direct and Indirect Effects of Low-Energy Electrons on DNA, Annu. Rev. Phys. Chem. 66 (2015) 379.

[9] K. Gokhberg, P. Kolorenč, A. I. Kuleff, L. S. Cederbaum, Siteand energy-selective slow-electron production through intermolecular Coulombic decay, Nature 505 (2014) 661.

[10] F. Trinter, M. S. Schöffler, H. K. Kim, F. P. Sturm, K. Cole, N. Neumann, A. Vredenborg, J. Williams, I. Bocharova, R. Guillemin, M. Simon, A. Belkacem, A. L. Landers, T. Weber, H. Schmidt-Böcking, R. Dörner, J. T., Resonant Auger decay driving intermolecular Coulombic decay in molecular dimers, Nature 505 (2014) 664.

[11] J. Zobeley, R. Santra, L. S. Cederbaum, Electronic decay in weakly bound heteroclusters: Energy transfer versus electron transfer, The Journal of Chemical Physics 115 (2001) 5076-5088.

[12] V. Averbukh, L. S. Cederbaum, Calculation of interatomic decay widths of vacancy states delocalized due to inversion symmetry, J. Chem. Phys. 125 (2006) 094107.

[13] S. Scheit, L. S. Cederbaum, H.-D. Meyer, Time-dependent interplay between electron emission and fragmentation in the interatomic Coulombic decay, J. Chem. Phys. 118 (2003) 2092.

[14] S. Scheit, V. Averbukh, H.-D. Meyer, N. Moiseyev, R. Santra, T. Sommerfeld, J. Zobeley, L. S. Cederbaum, On the interatomic Coulombic decay in the Ne dimer, J. Chem. Phys. 121 (2004) 8393.

[15] R. Santra, J. Zobeley, L. S. Cederbaum, Electronic decay of valence holes in clusters and condensed matter, Phys. Rev. B 64 (2001) 245104.

[16] D. C. Griffin, D. M. Mitnik, N. R. Badnell, Electron-impact excitation of $\mathrm{Ne}^{+}$, Journal of Physics B: Atomic, Molecular and Optical Physics 34 (2001) 4401.

[17] G. Öhrwall, M. Tchaplyguine, M. Lundwall, R. Feifel, H. Bergersen, T. Rander, A. Lindblad, J. Schulz, S. Peredkov, S. Barth, S. Marburger, U. Hergenhahn, S. Svensson, O. Björneholm, Femtosecond Interatomic Coulombic Decay in Free Neon Clusters: Large Lifetime Differences between Surface and Bulk, Phys. Rev. Lett. 93 (2004) 173401.

[18] M. Mucke, M. Braune, S. Barth, M. Förstel, T. Lischke, V. Ulrich, T. Arion, U. Becker, A. Bradshaw, U. Hergenhahn, A hitherto unrecognized source of low-energy electrons in water, Nature Physics 6 (2010) 143.

[19] T. Jahnke, H. Sann, T. Havermeier, K. Kreidi, C. Stuck, M. Meckel, M. Schöffler, N. Neumann, R. Wallauer, S. Voss, A. Czasch, O. Jagutzki, A. Malakzadeh, F. Afaneh, T. Weber, H. Schmidt-Böcking, R. Dörner, Ultrafast energy transfer between water molecules, Nature Physics 6 (2010) 139.

[20] S. Thürmer, M. Ončák, N. Ottosson, R. Seidel, U. Hergenhahn, S. E. Bradforth, P. Slavíček, B. Winter, On the nature and origin of dicationic, charge-separated species formed in liquid water on X-ray irradiation, Nature Chem. 5 (2013) 590.

[21] P. Slavíček, B. Winter, L. S. Cederbaum, K. N. V., Proton-transfer mediated enhancement of non-local electronic relaxation processes in X-ray irradiated liquid water, J. Am. Chem. Soc. 136 (2014) 18170.

[22] F. Trinter, J. B. Williams, M. Weller, M. Waitz, M. Pitzer, J. Voigtsberger, C. Schober, G. Kastirke, C. Müller, C. Goihl, P. Burzynski, F. Wiegandt, R. Wallauer, A. Kalinin, L. Schmidt, M. Schöffler, Y.-C. Chiang, K. Gokhberg, T. Jahnke, R. Dörner, Vibrationally resolved decay width of interatomic Coulombic decay in HeNe, Phys. Rev. Lett. 111 (2013) 233004.

[23] G. Jabbari, S. Klaiman, Y.-C. Chiang, F. Trinter, T. Jahnke, K. Gokhberg, $\mathrm{Ab}$ initio calculation of ICD widths in photoexcited HeNe, J. Chem. Phys. 
140 (2014) 224305

[24] A. Russek, W. Mehlhorn, Post-collision interaction and the Auger lineshape, Journal of Physics B: Atomic and Molecular Physics 19 (1986) 911.

[25] A. Niehaus, Analysis of post-collision interactions in Auger processes following near-threshold inner-shell photoionization, J. Phys. B 10 (1977) 1845 .

[26] F. Trinter, J. B. Williams, M. Weller, M. Waitz, M. Pitzer, J. Voigtsberger, C. Schober, G. Kastirke, C. Müller, C. Goihl, P. Burzynski, F. Wiegandt, T. Bauer, R. Wallauer, H. Sann, A. Kalinin, L. P. H. Schmidt, M. Schöffler, N. Sisourat, T. Jahnke, Evolution of Interatomic Coulombic Decay in the Time Domain, Phys. Rev. Lett. 111 (2013) 093401.

[27] K. Schnorr, A. Senftleben, M. Kurka, A. Rudenko, L. Foucar, G. Schmid, A. Broska, T. Pfeifer, K. Meyer, D. Anielski, R. Boll, D. Rolles, M. Kübel, M. F. Kling, Y. H. Jiang, S. Mondal, T. Tachibana, K. Ueda, T. Marchenko, M. Simon, G. Brenner, R. Treusch, S. Scheit, V. Averbukh, J. Ullrich, C. D. Schröter, R. Moshammer, Time-Resolved Measurement of Interatomic Coulombic Decay in $\mathrm{Ne}_{2}$, Phys. Rev. Lett. 111 (2013) 093402.

[28] P. Emma, R. Akre, J. Arthur, R. Bionta, C. Bostedt, J. Bozek, A. Brachmann, P. Bucksbaum, R. Coffee, F.-J. Decker, Y. Ding, D. Dowell, S. Edstrom, A. Fisher, J. Frisch, S. Gilevich, J. Hastings, G. Hays, P. Hering, Z. Huang, R. Iverson, H. Loos, M. Messerschmidt, A. Miahnahri, S. Moeller, H.-D. Nuhn, G. Pile, D. Ratner, J. Rzepiela, D. Schultz, T. Smith, P. Stefan, H. Tompkins, J. Turner, J. Welch, W. White, J. Wu, G. Yocky, J. Galayda, First lasing and operation of an angstromwavelength free-electron laser, Nat. Photon. 4 (2010) 641-647.

[29] M. Harmand, R. Coffee, M. Bionta, M. Chollet, D. French, D. Zhu, D. Fritz, T. Lemke, N. Medvedev, B. Ziaja, S. Toleikis, M. Cammarata, Achieving few-femtosecond time-sorting at hard X-ray freeelectron lasers, Nature Photon. 7 (2013) 215-218.

[30] Y. Ovcharenko, V. Lyamayev, R. Katzy, M. Devetta, A. LaForge, O. Plekan, P. Finetti, M. Di Fraia, M. Mudrich, M. Krikunova, P. Piseri, M. Coreno, N. Brauer, T. Mazza, S. Stranges, C. Grazioli, R. Richter, K. C. Prince, M. Drabbels, C. Callegari, F. Stienkemeier, Novel collective autoionization process observed in electron spectra of He clusters, Phys. Rev. Lett. 112 (2014) 073401.

[31] A. C. LaForge, M. Drabbels, N. B. Brauer, M. Coreno, M. Devetta, M. Di Fraia, P. Finetti, C. Grazioli, R. Katzy, V. Lyamayev, T. Mazza, M. Mudrich, P. O'Keeffe, Y. Ovcharenko, P. Piseri, O. Plekan, K. C. Prince, R. Richter, S. Stranges, C. Callegari, M. T., F. Stienkemeier, Collective Autoionization in Multiply-Excited Systems: A novel ionization process observed in Helium Nanodroplets, Sci. Rep. 4 (2014) 3621.

[32] W. Ackermann, G. Asova, V. Ayvazyan, A. Azima, N. Baboi, J. Bahr, V. Balandin, B. Beutner, A. Brandt, A. Bolzmann, R. Brinkmann, O. I. Brovko, M. Castellano, P. Castro, L. Catani, E. Chiadroni, S. Choroba, A. Cianchi, J. T. Costello, D. Cubaynes, J. Dardis, W. Decking, H. Delsim-Hashemi, A. Delserieys, G. D. Pirro, M. Dohlus, S. Dusterer, A. Eckhardt, H. T. Edwards, B. Faatz, J. Feldhaus, K. Flottmann, J. Frisch, L. Frohlich, T. Garvey, U. Gensch, C. Gerth, M. Gorler, N. Golubeva, H.-J. Grabosch, M. Grecki, O. Grimm, K. Hacker, U. Hahn, J. H. Han, K. Honkavaara, T. Hott, M. Huning, Y. Ivanisenko, E. Jaeschke, W. Jalmuzna, T. Jezynski, R. Kammering, V. Katalev, K. Kavanagh, E. T. Kennedy, S. Khodyachykh, K. Klose, V. Kocharyan, M. Korfer, M. Kollewe, W. Koprek, S. Korepanov, D. Kostin, M. Krassilnikov, G. Kube, M. Kuhlmann, C. L. S. Lewis, L. Lilje, T. Limberg, D. Lipka, F. Lohl, H. Luna, M. Luong, M. Martins, M. Meyer, P. Michelato, V. Miltchev, W. D. Moller, L. Monaco, W. F. O. Muller, O. Napieralski, O. Napoly, P. Nicolosi, D. Nolle, T. Nunez, A. Oppelt, C. Pagani, R. Paparella, N. Pchalek, J. Pedregosa-Gutierrez, B. Petersen, B. Petrosyan, G. Petrosyan, L. Petrosyan, J. Pfluger, E. Plonjes, L. Poletto, K. Pozniak, E. Prat, D. Proch, P. Pucyk, P. Radcliffe, H. Redlin, K. Rehlich, M. Richter, M. Roehrs, J. Roensch, R. Romaniuk, M. Ross, J. Rossbach, V. Rybnikov, M. Sachwitz, E. L. Saldin, W. Sandner, H. Schlarb, B. Schmidt, M. Schmitz, P. Schmuser, J. R. Schneider, E. A. Schneidmiller, S. Schnepp, S. Schreiber, M. Seidel, D. Sertore, A. V. Shabunov, C. Simon, S. Simrock, E. Sombrowski, A. A. Sorokin, P. Spanknebel, R. Spesyvtsev, L. Staykov, B. Steffen, F. Stephan, F. Stulle, H. Thom, K. Tiedtke, M. Tischer, S. Toleikis, R. Treusch, D. Trines, I. Tsakov, E. Vogel, T. Weiland, H. Weise, M. Wellhofer, M. Wendt, I. Will, A. Winter, K. Wittenburg, W. Wurth, P. Yeates, M. V. Yurkov, I. Zagorodnov,
K. Zapfe, Operation of a free-electron laser from the extreme ultraviolet to the water window, Nat. Photon. 1 (2007) 336-342.

[33] J. Ullrich, R. Moshammer, A. Dorn, R. Dörner, L. P. H. Schmidt, H. Schmidt-Böcking, Recoil-ion and electron momentum spectroscopy: reaction-microscopes, Reports on Progress in Physics 66 (2003) 1463.

[34] S. Düsterer, P. Radcliffe, G. Geloni, U. Jastrow, M. Kuhlmann, E. Plönjes, K. Tiedtke, R. Treusch, J. Feldhaus, P. Nicolosi, L. Poletto, P. Yeates, H. Luna, J. T. Costello, P. Orr, D. Cubaynes, M. Meyer, Spectroscopic characterization of vacuum ultraviolet free electron laser pulses, Opt. Lett. 31 (2006) 1750-1752.

[35] A. van Deursen, J. Reuss, Molecular beam intensities and collision crosssections of small $\mathrm{Ne}, \mathrm{H}_{2}, \mathrm{~N}_{2}, \mathrm{NO}$ and $\mathrm{O}_{2}$ clusters, International Journal of Mass Spectrometry and Ion Physics 23 (1977) 109-122.

[36] S. D. Stoychev, A. I. Kuleff, F. Tarantelli, L. S. Cederbaum, On the interatomic electronic processes following Auger decay in neon dimer, J. Chem. Phys. 129 (2008) 074307.

[37] T. Jahnke, A. Czasch, M. Schöffler, S. Schössler, M. Käsz, J. Titze, K. Kreidi, R. E. Grisenti, A. Staudte, O. Jagutzki, L. Schmidt, S. K. Semenov, N. A. Cherepkov, H. Schmidt-Böcking, R. Dörner, Photoelectron and ICD electron angular distributions from fixed-in-space neon dimers, J. Phys. B: Atomic, Molecular and Optical Physics 40 (2007) 2597.

[38] S. Scheit, private communication, 2013.

[39] K. Meyer, C. Ott, P. Raith, A. Kaldun, Y. Jiang, A. Senftleben, M. Kurka, R. Moshammer, J. Ullrich, T. Pfeifer, Noisy Optical Pulses Enhance the Temporal Resolution of Pump-Probe Spectroscopy, Phys. Rev. Lett. 108 (2012) 098302.

[40] T. Pfeifer, Y. H. Jiang, S. Düsterer, R. Moshammer, J. Ullrich, Partialcoherence method to model experimental free-electron laser pulse statistics, Opt. Lett. 35 (2010) 3441-3443.

[41] R. Santra, L. S. Cederbaum, Coulombic Energy Transfer and Triple Ionization in Clusters, Phys. Rev. Lett. 90 (2003) 153401.

[42] K. Kreidi, P. V. Demekhin, T. Jahnke, T. Weber, T. Havermeier, X.-J. Liu, Y. Morisita, S. Schössler, L. P. H. Schmidt, M. Schöffler, M. Odenweller, N. Neumann, L. Foucar, J. Titze, B. Ulrich, F. Sturm, C. Stuck, R. Wallauer, S. Voss, I. Lauter, H. K. Kim, M. Rudloff, H. Fukuzawa, G. Prümper, N. Saito, K. Ueda, A. Czasch, O. Jagutzki, H. SchmidtBöcking, S. Scheit, L. S. Cederbaum, R. Dörner, Photo- and AugerElectron Recoil Induced Dynamics of Interatomic Coulombic Decay, Phys. Rev. Lett. 103 (2009) 033001.

[43] V. Averbukh, I. B. Müller, L. S. Cederbaum, Mechanism of Interatomic Coulombic Decay in Clusters, Phys. Rev. Lett. 93 (2004) 263002.

[44] J. Yeh, I. Lindau, Atomic subshell photoionization cross sections and asymmetry parameters: $1 \leq \mathrm{Z} \leq 103$, Atomic Data and Nuclear Data Tables 32 (1985) 1-155.

[45] R. Santra, L. S. Cederbaum, An efficient combination of computational techniques for investigating electronic resonance states in molecules, J. Chem. Phys. 115 (2001) 6853.

[46] N. Vaval, L. S. Cederbaum, Ab initio lifetimes in the interatomic Coulombic decay of neon clusters computed with propagators, J. Chem. Phys. 126 (2007) 164110.

[47] V. Averbukh, P. V. Demekhin, P. Kolorenč, S. Scheit, S. D. Stoychev, A. I. Kuleff, Y.-C. Chiang, K. Gokhberg, S. Kopelke, N. Sisourat, L. S. Cederbaum, Interatomic electronic decay processes in singly and multiply ionized clusters, J. Electron Spectrosc. Relat. Phenom. 183 (2011) 36.

[48] R. Kienberger, E. Goulielmakis, M. Uiberacker, A. Baltuska, V. Yakovlev, F. Bammer, A. Scrinzi, T. Westerwalbesloh, U. Kleineberg, U. Heinzmann, M. Drescher, F. Krausz, Atomic transient recorder, Nature 427 (2004) 817-821.

[49] M. Schultze, M. Fie, N. Karpowicz, J. Gagnon, M. Korbman, M. Hofstetter, S. Neppl, A. L. Cavalieri, Y. Komninos, T. Mercouris, C. A. Nicolaides, R. Pazourek, S. Nagele, J. Feist, J. Burgdörfer, A. M. Azzeer, R. Ernstorfer, R. Kienberger, U. Kleineberg, E. Goulielmakis, F. Krausz, V. S. Yakovlev, Delay in Photoemission, Science 328 (2010) 1658-1662. 